\title{
A Hölder-Young-Lieb inequality for norms of Gaussian Wick products
}

\author{
Paolo Da Pelo ${ }^{1}$, Alberto Lanconelli ${ }^{1}$ and Aurel I. Stan ${ }^{2}$ \\ ${ }^{1}$ Dipartimento di Matematica \\ ${ }^{1}$ Dipartimento di Matematica \\ Universita' degli Studi di Bari \\ Via E. Orabona, 4 \\ 70125 Bari - Italia \\ Universita' degli Studi di Bari \\ Via E. Orabona, 4 \\ 70125 Bari - Italia \\ E-mail: dapelo@dm.uniba.it \\ E-mail:lanconelli@dm.uniba.it \\ ${ }^{2}$ Department of Mathematics \\ Ohio State University at Marion \\ 1465 Mount Vernon Avenue \\ Marion, OH 43302, U.S.A. \\ E-mail: stan.7@osu.edu
}

\begin{abstract}
An important connection between the finite dimensional Gaussian Wick product and Lebesgue convolution product will be proven first. Then this connection will be used to prove an important Hölder inequality for the norms of Gaussian Wick products, reprove Nelson hypercontractivity inequality, and prove a more general inequality whose marginal cases are the Hölder and Nelson inequalities mentioned before. We will show that there is a deep connection between the Gaussian Hölder inequality and classic Hölder inequality, between the Nelson hypercontractivity and classic Young inequality with the sharp constant, and between the third more general inequality and an extension by Lieb of the Young inequality with the best constant. Since the Gaussian probability measure exists even in the infinite dimensional case, the above three inequalities can be extended, via a classic Fatou's lemma argument, to the infinite dimensional framework.
\end{abstract}

Key words and phrases: Wick product, second quantization operator, convolution product, Hölder inequality, Young inequality, Lieb inequality, exponential functions.

AMS 2000 classification: 44A35, 60H40, 60H10.

\section{Introduction}

If $(\Omega, \mathcal{F}, P)$ is a probability space and $H$ is a closed subspace of $L^{2}(\Omega, \mathcal{F}, P)$, such that every element $h$ from $H$ is normally distributed, with mean zero, then $H$ is called a (centered) Gaussian Hilbert space. If $H$ is a separable Gaussian Hilbert space and $\mathcal{F}(H)$ denotes the 
smallest sub-sigma algebra of $\mathcal{F}$, with respect to which all random variables $h$ from $H$ are measurable, then it was proven in [7] that for any two complex-valued functions $\varphi$ and $\psi$ in $L^{2}(\Omega, \mathcal{F}(H), P)$, and any two positive numbers $u$ and $v$, such that $(1 / u)+(1 / v)=1$, the Wick product of $\Gamma((1 / \sqrt{u}) I) \varphi$ and $\Gamma((1 / \sqrt{v} I)) \psi$, denoted by $\Gamma((1 / \sqrt{u}) I) \varphi \diamond \Gamma((1 / \sqrt{v}) I) \psi$, belongs to $L^{2}(\Omega, \mathcal{F}(H), P)$ and the following inequality holds:

$$
\left\|\Gamma\left(\frac{1}{\sqrt{u}} I\right) \varphi \diamond \Gamma\left(\frac{1}{\sqrt{v}} I\right) \psi\right\|_{2} \leq\|\varphi\|_{2} \cdot\|\psi\|_{2},
$$

where $\Gamma(c I)$ denotes the second quantization operator of $c$ times the identity operator $I$, for any complex constant $c$, and $\|\cdot\|_{2}$ the $L^{2}$-norm. The proof was based on the orthogonal structure of the space $L^{2}(\Omega, \mathcal{F}(H), P)$ (Fock decomposition) and Cauchy-BuniakovskiSchwarz inequality. The authors of [7] regarded inequality (1.1) as a Young inequality for White Noise Analysis, thinking that the Wick product, in the Gaussian case, is an analogue of the convolution product, from the classic Fourier Analysis. However, after discussing with other mathematicians, they were convinced that this inequality should be called a Hölder inequality for White Noise Analysis, since the Wick product is an analogue of the classic product of two series.

The Wick product can be defined not only in the Gaussian case, but also for any probability measure $\mu$ on $\mathbb{R}$ having finite moments of all orders. In [8] it was proven that for any probability measure $\mu$, that is not a delta measure (that means whose support does not reduce to a single point), if $r \geq 2$ is a fixed number, and $u$ and $v$ are positive numbers, such that, the inequality

$$
\left\|\Gamma\left(\frac{1}{\sqrt{u}} I\right) \varphi \diamond \Gamma\left(\frac{1}{\sqrt{v}} I\right) \psi\right\|_{r} \leq\|\varphi\|_{r} \cdot\|\psi\|_{r},
$$

holds for any $\varphi$ and $\psi$ in $L^{r}(\mathbb{R}, \mu)$, we must have:

$$
\frac{1}{u}+\frac{1}{v} \leq 1
$$

Therefore, if one can prove inequality (1.1), then it is the best inequality that he (she) can get not only in the Gaussian case, but also for all non-trivial probability measures.

In [9] it was proven, in the Gaussian case, that if $u$ and $v$ are positive numbers, such that $(1 / u)+(1 / v)=1$, then any two real valued functions $\varphi$ and $\psi$ in $L^{1}(\Omega, \mathcal{F}(H), P)$, the Wick product of $\Gamma((1 / \sqrt{u}) I) \varphi \diamond \Gamma((1 / \sqrt{v}) I) \psi$ belongs to $L^{1}(\Omega, \mathcal{F}(H), P)$, and the following inequality holds:

$$
\left\|\Gamma\left(\frac{1}{\sqrt{u}} I\right) \varphi \diamond \Gamma\left(\frac{1}{\sqrt{v}} I\right) \psi\right\|_{1} \leq\|\varphi\|_{1} \cdot\|\psi\|_{1} .
$$

In [9] it was also proven, in the Gaussian case, that for any two real valued functions $\varphi$ and $\psi$ in $L^{\infty}(\Omega, \mathcal{F}(H), P)$, and any two positive numbers $u$ and $v$, such that $(1 / u)+(1 / v)=1$, the Wick product of $\Gamma((1 / \sqrt{u}) I) \varphi \diamond \Gamma((1 / \sqrt{v}) I) \psi$ belongs to $L^{\infty}(\Omega, \mathcal{F}(H), P)$, and the following inequality holds:

$$
\left\|\Gamma\left(\frac{1}{\sqrt{u}} I\right) \varphi \diamond \Gamma\left(\frac{1}{\sqrt{v}} I\right) \psi\right\|_{\infty} \leq\|\varphi\|_{\infty} \cdot\|\psi\|_{\infty} .
$$


To prove the inequalities (1.4) and (1.5), the authors proved first a Jensen inequality for Gaussian Wick products, inspired by the Jensen inequality from [5].

In this paper, we will prove first a lemma that connects the Gaussian Wick product to the classic convolution product of the Lebesgue measure. We will then use this lemma to prove the following inequalities.

Let $p \in[1, \infty]$. If $u$ and $v$ are positive numbers, such that $(1 / u)+(1 / v)=1$, then for any two complex valued functions $\varphi$ and $\psi$ in $L^{p}(\Omega, \mathcal{F}(H), P)$, the Wick product $\Gamma((1 / \sqrt{u}) I) \varphi \diamond$ $\Gamma((1 / \sqrt{v}) I) \psi$ belongs to $L^{p}(\Omega, \mathcal{F}(H), P)$, and the following inequality holds:

$$
\left\|\Gamma\left(\frac{1}{\sqrt{u}} I\right) \varphi \diamond \Gamma\left(\frac{1}{\sqrt{v}} I\right) \psi\right\|_{p} \leq\|\varphi\|_{p} \cdot\|\psi\|_{p} .
$$

We will show that via the lemma connecting the Gaussian Wick product to the Lebesgue convolution product, inequality (1.6) reduces to the classic Hölder inequality.

Let $1<p \leq r<\infty$. Then for any $\varphi$ in $L^{p}(\Omega, \mathcal{F}(H) P)$ and any $\psi$ in $L^{\infty}(\Omega, \mathcal{F}(H)$ $P), \Gamma(\sqrt{p-1} / \sqrt{r-1}) \varphi \diamond \Gamma(\sqrt{r-p} / \sqrt{r-1}) \psi$ belongs to $L^{r}(\Omega, \mathcal{F}(H) P)$ and the following inequality holds:

$$
\left\|\left[\Gamma\left(\frac{\sqrt{p-1}}{\sqrt{r-1}}\right) \varphi\right] \diamond\left[\Gamma\left(\frac{\sqrt{r-p}}{\sqrt{r-1}}\right) \psi\right]\right\|_{r} \leq\|\varphi\|_{p} \cdot\|\psi\|_{\infty} .
$$

In particular, if we choose $\psi=1$ (the constant random variable equal to 1 ), the we get the classic Nelson hypercontractivity inequality:

$$
\left\|\Gamma\left(\frac{\sqrt{p-1}}{\sqrt{r-1}}\right) \varphi\right\|_{r} \leq\|\varphi\|_{p}
$$

We will show that via the lemma connecting the Gaussian Wick product to the Lebesgue convolution product, this inequality reduces to the Young inequality with the best constant proven by Beckner and Brascamp-Lieb in [1] and [2], respectively.

Finally we will prove that if $u$ and $v$ are positive numbers, such that $(1 / u)+(1 / v)=1$, and $p, q$, and $r$ are in $[1, \infty]$, such that:

$$
\frac{1}{r-1}=\frac{1}{u(p-1)}+\frac{1}{v(q-1)}
$$

then for any $\varphi$ in $L^{p}(\Omega, \mathcal{F}(H), P)$ and any $\psi$ in $L^{q}(\Omega, \mathcal{F}(H), P), \Gamma((1 / \sqrt{u}) I) \varphi \diamond \Gamma((1 / \sqrt{v}) I) \psi$ belongs to $L^{r}(\Omega, \mathcal{F}(H), P)$, and the following inequality holds:

$$
\left\|\Gamma\left(\frac{1}{\sqrt{u}} I\right) \varphi \diamond \Gamma\left(\frac{1}{\sqrt{v}} I\right) \psi\right\|_{r} \leq\|\varphi\|_{p} \cdot\|\psi\|_{q} .
$$

We will show that via the lemma connecting the Gaussian Wick product to the Lebesgue convolution product, this inequality is connected to the fully generalized Young's inequality proven by Lieb in [10]. See also page 100 of [11].

It is easy to see that (1.6) and (1.7) are particular cases of (1.10), namely, (1.10) reduces to (1.6) in the particular case $p=q=r$, and (1.10) reduces to (1.7) when $q=\infty$. In fact, condition (1.9) tells us that $1 /(r-1)$ is a convex combination of $1 /(p-1)$ and $1 /(q-1)$, and so, if we assume that $p \leq q$, then $p \leq r \leq q$. If we fix the left-endpoint $p$, of the interval $[p$, $q$ ], and let the right-endpoint $q$ vary from $p$ to $\infty$, then inequalities (1.6) and (1.7) are the 
"marginal" cases of (1.10): $q=p$ and $q=\infty$, respectively. So, one might say that proving (1.10) makes the proofs of (1.6) and (1.7) superfluous. However, we prefer to prove first (1.6), then (1.7), and finally (1.10), to show how they are connected to the following important and deep inequalities of classical Analysis: Hölder, Young with the sharp constant, and Lieb. We will also see that as we move from inequality (1.6) to (1.10), the complexity of the proof increases more and more.

All the above inequalities are sharp, the equality occurring for some exponential functions.

In section 2, we present a short background of the theory of Gaussian Hilbert spaces. This background includes the definition of the Wick product and second quantization operator of a constant times the identity. In section 3 we prove the important lemma connecting the Gaussian Wick product to the Lebesgue convolution product. Finally in the last section we prove the main results of this paper.

\section{Background}

In this section we present a minimal background about Gaussian Wick products and second quantization operators. The frameworks in which this background can be presented are many. One can use, for example, Hida's White Noise Theory (see [6] or [12]), Malliavin Calculus, the multiple Wiener integrals, a Fock space, or the theory of Gaussian Hilbert Spaces. All of these theories are leading to the same notion of Wick product. Since we are not going to use generalized functions, we are going to use the theory of Gaussian Hilbert Spaces as outlined in [5].

Let $(\Omega, \mathcal{F}, P)$ be a probability space and $H$ a closed subspace of $L^{2}(\Omega, \mathcal{F}, P)$, such that every element $h$ of $H$ is normally distributed with mean zero. We call $H$ a (centered) Gaussian Hilbert space. We assume that $H$ is separable. For all non-negative integers $n$, we define the space:

$$
F_{n}=\left\{f\left(h_{1}, \ldots, h_{d}\right) \mid d \geq 1, h_{i} \in H, i=1, \ldots, d, f \text { is polynomial, } \operatorname{deg}(f) \leq n\right\},
$$

where $\operatorname{deg}(f)$ denotes the degree of the polynomial $f$. Since the Gaussian random variables have finite moments of all orders, each space $F_{n}$ is contained in $L^{2}(\Omega, \mathcal{F}, P)$. We have:

$$
\mathbb{C}=F_{0} \subset F_{1} \subset F_{2} \subset \cdots \subset L^{2}(\Omega, \mathcal{F}, P) .
$$

We define now the following spaces: $G_{0}:=F_{0}$ and for all $n \geq 1$,

$$
G_{n}:=\bar{F}_{n} \ominus \bar{F}_{n-1},
$$

where $\bar{F}$ denotes the closure of $F$ in $L^{2}(\Omega, \mathcal{F}, P)$, for any subspace $F$ of $L^{2}(\Omega, \mathcal{F}, P)$. For each non-negative integer $n$, we call $G_{n}$ the $n$-th homogenous chaos space generated by $H$, and every element $\varphi$ from $G_{n}$, a homogenous polynomial random variable of degree $n$. We define the following Hilbert space:

$$
\mathcal{H}=\oplus_{n=0}^{\infty} G_{n}
$$

and call it the chaos space generated by $H$. Let us observe that every random variable from $\mathcal{H}$ is measurable with respect to the sigma-algebra $\mathcal{F}(H)$ generated by the elements $h$ from $H$. The reciprocal is also true and the following theorem holds (see Theorem 2.6., from page 18 , in [5]). 


\section{Theorem 2.1}

$$
\mathcal{H}=L^{2}(\Omega, \mathcal{F}(H), P)
$$

From now on, because of Theorem [2.1, whatever random variables we will consider, they will be measurable with respect to $\mathcal{F}(H)$.

For every $n \geq 0$, we denote by $P_{n}$ the orthogonal projection from $\mathcal{H}$ onto $G_{n}$. The Wick product is defined first for any two homogenous polynomial random variables, and then extended in a bilinear way, as explained below. For any non-negative integers $m$ and $n$, and any $\varphi$ in $G_{m}$ and $\psi$ in $G_{n}$, we define:

$$
\varphi \diamond \psi=P_{m+n}(\varphi \cdot \psi) .
$$

If $\varphi=\sum_{n=0}^{\infty} f_{n} \in \mathcal{H}$ and $\psi=\sum_{n=0}^{\infty} g_{n} \in \mathcal{H}$, where $f_{n}$ and $g_{n}$ are in $G_{n}$, for all $n \geq 0$, then the Wick product of $\varphi$ and $\psi$, denoted by $\varphi \diamond \psi$, is defined as:

$$
\varphi \diamond \psi=\sum_{k=0}^{\infty}\left[\sum_{p+q=k}\left(f_{p} \diamond g_{q}\right)\right] .
$$

Of course, there might be problems with the convergence, in the $L^{2}$-sense, of the series from the right-hand side of (2.3), but at least for the case when $\varphi$ and $\psi$ are polynomial random variables (i.e., only finitely many $f_{n}$ and $g_{n}, n \geq 1$, are different from zero), the Wick product $\varphi \diamond \psi$ is well-defined.

If $c$ is a fixed complex number, then we define the second quantization operator of $c I$, where $I$ denotes the identity operator of $H$, by:

$$
\Gamma(c I) \varphi=\sum_{n=0}^{\infty} c^{n} f_{n}
$$

for all $\varphi=\sum_{n=0}^{\infty} f_{n} \in \mathcal{H}$, where $f_{n}$ is in $G_{n}$, for all $n \geq 0$. From now we are going to drop the letter $I$, and write simply $\Gamma(c)$ instead of $\Gamma(c I)$. It is clear, that if $|c| \leq 1$, we have $\Gamma(c) \varphi \in \mathcal{H}$, and the following inequality holds:

$$
\|\Gamma(c) \varphi\|_{2} \leq\|\varphi\|_{2}
$$

where $\|\cdot\|_{2}$ denotes the $L^{2}$-norm. Moreover, as it is shown in [5], Theorem 4.12, page 48, if $c$ is real and $|c| \leq 1$, then the second quantization operator $\Gamma(c)$ has a unique continuous extension from $L^{1}(\Omega, \mathcal{F}(H), P)$ to $L^{1}(\Omega, \mathcal{F}(H), P)$, that we denote also by $\Gamma(c)$, and this extension is a bounded linear operator, of operatorial norm equal to 1 , from $L^{p}(\Omega, \mathcal{F}(H)$, $P)$ to $L^{p}(\Omega, \mathcal{F}(H), P)$, for all $1 \leq p \leq \infty$.

The second quantization operators are distributive with respect to the Wick product, in the following sense:

Lemma 2.2 For all $c$ in $\mathbb{C}$, such that $|c| \leq 1$, and all $\varphi$ and $\psi$ in $L^{2}(\Omega, \mathcal{F}(H)$, $P)$, such that $\varphi \diamond \xi$ belongs to $L^{2}(\Omega, \mathcal{F}(H), P)$, we have:

$$
\Gamma(c)(\varphi \diamond \psi)=\Gamma(c) \varphi \diamond \Gamma(c) \psi .
$$


The second quantization operators are very important in assuring the convergence of the Wick product of two random variables, due to the following theorems, from [7] and [9], respectively.

Theorem 2.3 For any $u$ and $v$ positive numbers, such that $(1 / u)+(1 / v)=1$, and for any $\varphi$ and $\psi$ in $L^{2}(\Omega, \mathcal{F}(H), P)$, the Wick product $\Gamma(1 / \sqrt{u}) \varphi \diamond \Gamma(1 / \sqrt{v}) \psi$ belongs to $L^{2}(\Omega, \mathcal{F}(H)$, $P)$, and the following inequality holds:

$$
\left\|\Gamma\left(\frac{1}{\sqrt{u}}\right) \varphi \diamond \Gamma\left(\frac{1}{\sqrt{v}}\right) \psi\right\|_{2} \leq\|\varphi\|_{2} \cdot\|\psi\|_{2} .
$$

Theorem 2.4 For any $u$ and $v$ positive numbers, such that $(1 / u)+(1 / v)=1$, the bilinear operator $T_{u, v}: L^{2}(\Omega, \mathcal{F}(H), P) \times L^{2}(\Omega, \mathcal{F}(H), P) \rightarrow L^{2}(\Omega, \mathcal{F}(H), P)$, defined as:

$$
T_{u, v}(\varphi, \psi)=\Gamma\left(\frac{1}{\sqrt{u}}\right) \varphi \diamond \Gamma\left(\frac{1}{\sqrt{v}}\right) \psi,
$$

for any $\varphi$ and $\psi$ in $L^{2}(\Omega, \mathcal{F}(H), P)$, admits a unique continuous linear extension $\tilde{T}_{u, v}$ from $L^{1}(\Omega, \mathcal{F}(H), P) \times L^{1}(\Omega, \mathcal{F}(H), P)$ to $L^{1}(\Omega, \mathcal{F}(H), P)$. Moreover, if $\varphi$ and $\psi$ are real valued, then we have:

$$
\left\|\Gamma\left(\frac{1}{\sqrt{u}}\right) \varphi \diamond \Gamma\left(\frac{1}{\sqrt{v}}\right) \psi\right\|_{1} \leq\|\varphi\|_{1} \cdot\|\psi\|_{1}
$$

and

$$
\left\|\Gamma\left(\frac{1}{\sqrt{u}}\right) \varphi \diamond \Gamma\left(\frac{1}{\sqrt{v}}\right) \psi\right\|_{\infty} \leq\|\varphi\|_{\infty} \cdot\|\psi\|_{\infty} .
$$

In this paper, we will remove the condition "real valued" from the last sentence of the previous theorem.

There is an important family of random variables in this theory, that have some beautiful properties with respect to the Wick product and second quantization operators. These functions are called the (renormalized) exponential random variables, and are defined as follows. For any $\xi \in H_{c}$ (where $H_{c}$ denotes the complexification of $H$ ), we define the exponential function $\mathcal{E}_{\xi}$ generated by $\xi$, by the formula:

$$
\mathcal{E}_{\xi}:=\sum_{n=0}^{\infty} \frac{1}{n !} \xi^{\diamond n}
$$

where $\xi^{\diamond n}:=\xi \diamond \xi \diamond \cdots \diamond \xi$ ( $n$ times). As a random variable, $\mathcal{E}_{\xi}$ can be written as:

$$
\mathcal{E}_{\xi}(\omega)=e^{\xi(\omega)-\frac{1}{2}\langle\xi, \xi\rangle}
$$

for all $\omega \in \Omega$, where:

$$
\langle f, g\rangle:=E[f \cdot g]
$$

for all $f$ and $g$ in $H_{c}$, and $E$ denotes the expectation. It can be easily seen that $\mathcal{E}_{\xi}$ belongs to $L^{p}(\Omega, \mathcal{F}(H), P)$, for all $1 \leq p<\infty$, and all $\xi$ in $H_{c}$. The family of exponential functions is closed with respect to the Wick product and second quantization operators, as illustrated by the following lemma (see [6] and [12]). 
Lemma 2.5 For all $\xi$ and $\eta$ in $H_{c}$, and all $c$ in $\mathbb{C}$, we have:

$$
\mathcal{E}_{\xi} \diamond \mathcal{E}_{\eta}=\mathcal{E}_{\xi+\eta}
$$

and

$$
\Gamma(c) \mathcal{E}_{\xi}=\mathcal{E}_{c \xi}
$$

Finally, the exponential functions are important in defining the $S$-transform. If $\varphi \in L^{2}(\Omega$, $\mathcal{F}(H), P)$ and $\xi \in H_{c}$, then the $S$-transform of $\varphi$ at $\xi$ is defined by the formula:

$$
(S \varphi)(\xi)=E\left[\varphi \cdot \mathcal{E}_{\xi}\right] .
$$

The $S$-transform is a unitary operator from $L^{2}(\Omega, \mathcal{F}(H), P)$ onto a Hilbert space of holomorphic functions, which we are not going to describe, since it is not important in this paper. If one considers the $S$-transform as an analogue of Fourier or Laplace transforms from the classic Analysis, then the Wick product becomes automatically an analogue of the convolution product, due to the following easy to check property.

Lemma 2.6 For any two functions $\varphi$ and $\psi$ in $L^{2}(\Omega, \mathcal{F}(H), P)$, such that $\varphi \diamond \psi$ belongs to $L^{2}(\Omega, \mathcal{F}(H), P)$, we have:

$$
S(\varphi \diamond \psi)=(S \varphi) \cdot(S \psi)
$$

\section{An connection between the Gaussian Wick product and Lebesgue convolution product}

Let $d$ be a fixed positive integer. Let $d_{N} x$ denote the normalized Lebesgue measure on $\mathbb{R}^{d}$, $(1 / \sqrt{2 \pi})^{d} d x$, and $\mu$ the standard Gaussian probability measure on $\mathbb{R}^{d}$, i.e., $d \mu=e^{-\langle x, x\rangle / 2} d_{N} x$. If $X_{i}: \Omega \rightarrow \mathbb{R}, i=\{1,2, \ldots, d\}$ are independent standard normal random variables, and $\mathcal{F}$ is the sigma-algebra generated by them, then any random variable $Y: \Omega \rightarrow \mathbb{C}$, that is measurable with respect $\mathcal{F}$, can be written as $Y=g\left(X_{1}, X_{2}, \ldots, X_{d}\right)$, where $g: \mathbb{R}^{d} \rightarrow \mathbb{C}$ is a Borel measurable function. From now on we will write $g(x)$ with a lower case $x$ instead of the upper case $X$, and do the computations of integrals in terms of the probability distribution $\mu$ of $X$, where $X:=\left(X_{1}, X_{2}, \ldots, X_{d}\right)$. Observe that in terms of distributions, for any $p \geq 1$, the $L^{p}$ norm of a function $f(x)$ with respect to the Gaussian measure $\mu$ is the same as the $L^{p}$ norm of $f(x) e^{-\langle x, x\rangle /(2 p)}$ with respect to the normalized Lebesgue measure $d_{N} x$. This simple fact will be used throughout this paper. Everything will be done using the normalized Lebesgue measure. Throughout this paper, for any $p \in[1, \infty]$, we will denote by $\|\cdot\|_{p}$ and $\||\cdot|\|_{p}$ the $L^{p}$ norms with respect to the Gaussian measure $\mu$ and normalized Lebesgue measure $d_{N} x$, respectively.

We are now presenting a connection between the Gaussian Wick product $\diamond$ and convolution product $\star$ with respect to the normalized Lebesgue measure.

Lemma 3.1 Let $u$ and $v$ be positive numbers, such that: $(1 / u)+(1 / v)=1$. Then for any $\varphi$ and $\psi$ in $L^{1}\left(\mathbb{R}^{d}, \mu\right)$, we have that $\Gamma(1 / \sqrt{u}) \varphi \diamond \Gamma(1 / \sqrt{v}) \psi$ belongs to $L^{1}\left(\mathbb{R}^{d}, \mu\right)$ and:

$$
\begin{aligned}
& {\left[\varphi\left(\frac{1}{\sqrt{v}} x\right) e^{-\frac{\langle x, x\rangle}{2 v}}\right] \star\left[\psi\left(\frac{1}{\sqrt{u}} x\right) e^{-\frac{\langle x, x\rangle}{2 u}}\right] } \\
= & {\left[\Gamma\left(\frac{1}{\sqrt{u}}\right) \varphi \diamond \Gamma\left(\frac{1}{\sqrt{v}}\right) \psi\right]\left(\frac{1}{\sqrt{u v}} x\right) e^{-\frac{1}{2 u v}\langle x, x\rangle}, }
\end{aligned}
$$


where the convolution product in the left-hand side is computed with respect to the normalized Lebesgue measure $d_{N} x$.

We prove first an easier version of this lemma.

Lemma 3.2 Let $\mathcal{E}=\left\{\sum_{i=1}^{n} c_{i} e^{\left\langle\xi_{i}, x\right\rangle-(1 / 2)\left\langle\xi_{i}, \xi_{i}\right\rangle} \mid n \in \mathbb{N}, c_{i} \in \mathbb{C}, \xi_{i} \in \mathbb{C}^{d}, \forall i \in\{1,2, \ldots, n\}\right\}$. Let $u$ and $v$ be positive numbers, such that $(1 / u)+(1 / v)=1$. For any $\varphi$ and $\psi$ in $\mathcal{E}$, we have:

$$
\begin{aligned}
& \left\{[\Gamma(\sqrt{u}) \varphi]\left(\frac{x}{\sqrt{v}}\right) e^{-\frac{\langle x, x\rangle}{2 v}}\right\} \star\left\{[\Gamma(\sqrt{v}) \psi]\left(\frac{x}{\sqrt{u}}\right) e^{-\frac{\langle x, x\rangle}{2 u}}\right\} \\
= & (\varphi \diamond \psi)\left(\frac{x}{\sqrt{u v}}\right) e^{-\frac{\langle x, x\rangle}{2 u v}} .
\end{aligned}
$$

In particular, replacing $\varphi$ and $\psi$ by $\Gamma(1 / \sqrt{u}) \varphi$ and $\Gamma(1 / \sqrt{v}) \psi$, respectively, we obtain that (3.1) holds for any two functions that are linear combinations of exponential functions.

Proof. Since both sides of (3.2) are bilinear with respect to $\varphi$ and $\psi$, it is enough to check the relation for $\varphi(x)=e^{\langle\xi, x\rangle-(1 / 2)\langle\xi, \xi\rangle}$ and $\psi(x)=e^{\langle\eta, x\rangle-(1 / 2)\langle\eta, \eta\rangle}$, where $\xi$ and $\eta$ are arbitrarily fixed vectors in $\mathbb{C}^{d}$. Indeed, for these functions we have:

$$
\begin{aligned}
& \left\{[\Gamma(\sqrt{u}) \varphi]\left(\frac{\cdot}{\sqrt{v}}\right) e^{-\frac{\langle\cdot, \cdot\rangle}{2 v}}\right\} \star\left\{[\Gamma(\sqrt{v}) \psi]\left(\frac{\cdot}{\sqrt{u}}\right) e^{-\frac{\langle\cdot, \cdot\rangle}{2 u}}\right\}(x) \\
= & {\left[e^{\frac{\sqrt{u}}{\sqrt{v}}\langle\xi, \cdot\rangle-\frac{u}{2}\langle\xi, \xi\rangle-\frac{\langle\cdot, \cdot}{2 v}}\right] \star\left[e^{\frac{\sqrt{v}}{\sqrt{u}}\langle\eta, \cdot\rangle-\frac{v}{2}\langle\eta, \eta\rangle-\frac{\langle\cdot, \cdot\rangle}{2 u}}\right](x) } \\
= & \int_{\mathbb{R}^{d}} e^{\frac{\sqrt{u}}{\sqrt{v}}\langle\xi, x-y\rangle-\frac{u}{2}\langle\xi, \xi\rangle-\frac{\langle x-y, x-y\rangle}{2 v}} \cdot e^{\frac{\sqrt{v}}{\sqrt{u}}\langle\eta, y\rangle-\frac{v}{2}\langle\eta, \eta\rangle-\frac{\langle y, y\rangle}{2 u}} d_{N} y \\
= & e^{\frac{\sqrt{u}}{\sqrt{v}}\langle\xi, x\rangle-\frac{u}{2}\langle\xi, \xi\rangle-\frac{v}{2}\langle\eta, \eta\rangle-\frac{1}{2 v}\langle x, x\rangle} \int_{\mathbb{R}^{d}} e^{-\frac{1}{2}\langle y, y\rangle+\left\langle-\frac{\sqrt{u}}{\sqrt{v}} \xi+\frac{\sqrt{v}}{\sqrt{u}} \eta+\frac{1}{v} x, y\right\rangle} d_{N} y .
\end{aligned}
$$

We now perform the classic trick of completing the square, in the exponential, by subtracting and adding $(1 / 2)\langle-(\sqrt{u} / \sqrt{v}) \xi+(\sqrt{v} / \sqrt{u}) \eta+(1 / v) x,-(\sqrt{u} / \sqrt{v}) \xi+(\sqrt{v} / \sqrt{u}) \eta+(1 / v) x\rangle$, a factor that does not depend on the variable of integration $y$, and can be taken out of the integral. Thus, we obtain:

$$
\begin{aligned}
& \left\{[\Gamma(\sqrt{u}) \varphi]\left(\frac{\cdot}{\sqrt{v}}\right) e^{-\frac{\langle\cdot \cdot \cdot\rangle}{2 v}}\right\} \star\left\{[\Gamma(\sqrt{v}) \psi]\left(\frac{\cdot}{\sqrt{u}}\right) e^{-\frac{\langle\cdot \cdot \cdot\rangle}{2 u}}\right\}(x) \\
& =e^{\frac{\sqrt{u}}{\sqrt{v}}\langle\xi, x\rangle-\frac{u}{2}\langle\xi, \xi\rangle-\frac{v}{2}\langle\eta, \eta\rangle-\frac{1}{2 v}\langle x, x\rangle} \cdot e^{\frac{1}{2}\left\langle-\frac{\sqrt{u}}{\sqrt{v}} \xi+\frac{\sqrt{v}}{\sqrt{u}} \eta+\frac{1}{v} x,-\frac{\sqrt{u}}{\sqrt{v}} \xi+\frac{\sqrt{v}}{\sqrt{u}} \eta+\frac{1}{v} x\right\rangle} \\
& \times \int_{\mathbb{R}^{d}} e^{-\frac{1}{2}\left\langle y-\frac{\sqrt{u}}{\sqrt{v}} \xi+\frac{\sqrt{v}}{\sqrt{u}} \eta+\frac{1}{v} x, y-\frac{\sqrt{u}}{\sqrt{v}} \xi+\frac{\sqrt{v}}{\sqrt{u}} \eta+\frac{1}{v} x\right\rangle} d_{N} y \\
& =e^{-\frac{u}{2}\left(1-\frac{1}{v}\right)\langle\xi, \xi\rangle-\langle\xi, \eta\rangle-\frac{v}{2}\left(1-\frac{1}{u}\right)\langle\eta, \eta\rangle+\frac{\sqrt{u}}{\sqrt{v}}\left(1-\frac{1}{v}\right)\langle\xi, x\rangle+\frac{1}{\sqrt{u v}}\langle\eta, x\rangle-\frac{1}{2 v}\left(1-\frac{1}{v}\right)\langle x, x\rangle} \int_{\mathbb{R}^{d}} e^{-\frac{1}{2}\langle z, z\rangle} d_{N} z \\
& =e^{-\frac{u}{2} \cdot \frac{1}{u}\langle\xi, \xi\rangle-\langle\xi, \eta\rangle-\frac{v}{2} \cdot \frac{1}{v}\langle\eta, \eta\rangle+\frac{\sqrt{u}}{\sqrt{v}} \cdot \frac{1}{u}\langle\xi, x\rangle+\frac{1}{\sqrt{u v}} \cdot\langle\eta, x\rangle-\frac{1}{2 v} \cdot \frac{1}{u}\langle x, x\rangle} \cdot 1 \\
& =e^{-\frac{1}{2}\langle\xi+\eta, \xi+\eta\rangle+\left\langle\xi+\eta, \frac{1}{\sqrt{u v}} x\right\rangle-\frac{1}{2 u v} \cdot\langle x, x\rangle} \\
& =(\varphi \diamond \psi)\left(\frac{x}{\sqrt{u v}}\right) e^{-\frac{\langle x, x\rangle}{2 u v}} \text {, }
\end{aligned}
$$

since $(\varphi \diamond \psi)(x)=e^{\langle\xi+\eta, x\rangle-(1 / 2)\langle\xi+\eta, \xi+\eta\rangle}$. 
A complete proof of Lemma 3.1 is the following:

Proof. Since $\mathcal{E}$ is dense in $L^{1}\left(\mathbb{R}^{d}, \mu\right)$, there exist two sequences $\left\{f_{n}\right\}_{n \geq 1}$ and $\left\{g_{n}\right\}_{n \geq 1}$ of elements of $\mathcal{E}$, such that: $\left\|f_{n}-\varphi\right\|_{1} \rightarrow 0$ and $\left\|g_{n}-\psi\right\|_{1} \rightarrow 0$, as $n \rightarrow \infty$. For each $n \geq 1$, we have:

$$
\begin{aligned}
& {\left[f_{n}\left(\frac{x}{\sqrt{v}}\right) e^{-\frac{\langle x, x\rangle}{2 v}}\right] \star\left[g_{n}\left(\frac{x}{\sqrt{u}}\right) e^{-\frac{\langle x, x\rangle}{2 u}}\right] } \\
= & {\left[\Gamma\left(\frac{1}{\sqrt{u}}\right) f_{n} \diamond \Gamma\left(\frac{1}{\sqrt{v}}\right) g_{n}\right]\left(\frac{x}{\sqrt{u v}}\right) e^{-\frac{\langle x, x\rangle}{2 u v}} . }
\end{aligned}
$$

The left-hand side converges to $\left[\varphi\left(\frac{x}{\sqrt{v}}\right) e^{-\frac{\langle x, x\rangle}{2 v}}\right] \star\left[\psi\left(\frac{x}{\sqrt{u}}\right) e^{-\frac{\langle x, x\rangle}{2 u}}\right]$, in $L^{1}\left(\mathbb{R}^{d}, \mu\right)$, as $n \rightarrow \infty$, by Young inequality for the normalized Lebesgue measure. The right-hand side converges to $\left[\Gamma\left(\frac{1}{\sqrt{u}}\right) \varphi \diamond \Gamma\left(\frac{1}{\sqrt{v}}\right) \psi\right]\left(\frac{x}{\sqrt{u v}}\right) e^{-\frac{\langle x, x\rangle}{2 u v}}$, by Lanconelli-Stan inequality, from [9], about the $L^{1}$ norms for Wick products.

\section{Main results}

Theorem 4.1 (Finite dimensional Hölder inequality for Gaussian Wick products.) Let $d \in \mathbb{N}$ and $p \in[1, \infty]$ be fixed. Let $\mu$ denote the standard Gaussian probability measure on $\mathbb{R}^{d}$. Let $u$ and $v$ be positive numbers, such that $(1 / u)+(1 / v)=1$. Then for any $\varphi$ and $\psi$ in $L^{p}\left(\mathbb{R}^{d}, \mu\right), \Gamma(1 / \sqrt{u}) \varphi \diamond \Gamma(1 / \sqrt{v}) \psi \in L^{p}\left(\mathbb{R}^{d}, \mu\right)$ and the following inequality holds:

$$
\left\|\Gamma\left(\frac{1}{\sqrt{u}}\right) \varphi \diamond \Gamma\left(\frac{1}{\sqrt{v}}\right) \psi\right\|_{p} \leq\|\varphi\|_{p} \cdot\|\psi\|_{p} .
$$

Proof. Let $p^{\prime}$ be the conjugate of $p$, i.e., $(1 / p)+\left(1 / p^{\prime}\right)=1$. Let's multiply both sides of formula (3.1) by $e^{\langle x, x\rangle /\left(2 p^{\prime} u v\right)}$. We get:

$$
\begin{aligned}
& {\left[\Gamma\left(\frac{1}{\sqrt{u}}\right) \varphi \diamond \Gamma\left(\frac{1}{\sqrt{v}}\right) \psi\right]\left(\frac{x}{\sqrt{u v}}\right) e^{-\frac{\langle x, x\rangle}{2 u v}\left(1-\frac{1}{p^{\prime}}\right)} } \\
= & e^{\frac{\langle x, x\rangle}{2 p^{\prime} u v}}\left\{\left[\varphi\left(\frac{x}{\sqrt{v}}\right) e^{-\frac{\langle x, x\rangle}{2 v}}\right] \star\left[\psi\left(\frac{x}{\sqrt{u}}\right) e^{-\frac{\langle x, x\rangle}{2 u}}\right]\right\} \\
= & \int_{\mathbb{R}^{d}}\left[\varphi\left(\frac{x-y}{\sqrt{v}}\right) e^{-\frac{\langle x-y, x-y\rangle}{2 p v}}\right] e^{-\frac{\langle x-y, x-y\rangle}{2 p^{\prime} v}}\left[\psi\left(\frac{y}{\sqrt{u}}\right) e^{-\frac{\langle y, y\rangle}{2 p u}}\right] e^{-\frac{\langle y, y\rangle}{2 p^{\prime} u}} \cdot e^{\frac{\langle x, x\rangle}{2 p^{\prime} u v}} d_{N} y .
\end{aligned}
$$

Let $f(x):=\varphi((1 / \sqrt{v}) x) \exp (-[1 /(2 p v)]\langle x, x\rangle)$ and $g(x):=\psi((1 / \sqrt{u}) x) e(-[1 /(2 p u)]\langle x, x\rangle)$. With these notations we get:

$$
\begin{aligned}
& {\left[\Gamma\left(\frac{1}{\sqrt{u}}\right) \varphi \diamond \Gamma\left(\frac{1}{\sqrt{v}}\right) \psi\right]\left(\frac{x}{\sqrt{u v}}\right) e^{-\frac{\langle x, x\rangle}{2 u v} \cdot \frac{1}{p}} } \\
= & \int_{\mathbb{R}^{d}} f(x-y) g(y) e^{-\frac{1}{2 p}\left\langle y-\frac{1}{v} x, y-\frac{1}{v} x\right\rangle} d_{N} y .
\end{aligned}
$$


Putting the modulus in both sides, then introducing it in the integral in the right (triangle inequality), and then applying the Hölder inequality to the pair $\left(p, p^{\prime}\right)$, we get:

$$
\begin{aligned}
& \left|\left[\Gamma\left(\frac{1}{\sqrt{u}}\right) \varphi \diamond \Gamma\left(\frac{1}{\sqrt{v}}\right) \psi\right]\left(\frac{x}{\sqrt{u v}}\right) e^{-\frac{\langle x, x\rangle}{2 p u v}}\right| \\
\leq & {\left[\int_{\mathbb{R}^{d}}|f(x-y) g(y)|^{p} d_{N} y\right]^{\frac{1}{p}}\left[\int_{\mathbb{R}^{d}}\left(e^{-\frac{1}{2 p^{\prime}}\left\langle y-\frac{1}{v} x, y-\frac{1}{v} x\right\rangle}\right)^{p^{\prime}} d_{N} y\right]^{\frac{1}{p^{\prime}}} } \\
\leq & {\left[\int_{\mathbb{R}^{d}}|f(x-y)|^{p}|g(y)|^{p} d_{N} y\right]^{\frac{1}{p}} \cdot 1 . }
\end{aligned}
$$

Let us first raise the last inequality to the power $p$, then integrate it with respect to $x$, and apply Fubini (actually Tonelli) theorem. We obtain:

$$
\begin{aligned}
& \int_{\mathbb{R}^{d}}\left|\left[\Gamma\left(\frac{1}{\sqrt{u}}\right) \varphi \diamond \Gamma\left(\frac{1}{\sqrt{v}}\right) \psi\right]\left(\frac{x}{\sqrt{u v}}\right) e^{-\frac{\langle x, x\rangle}{2 p u v}}\right|^{p} d_{N} x \\
\leq & \int_{\mathbb{R}^{d}}\left[\int_{\mathbb{R}^{d}}|f(x-y)|^{p}|g(y)|^{p} d_{N} y\right] d_{N} x \\
= & \int_{\mathbb{R}^{d}}|g(y)|^{p}\left[\int_{\mathbb{R}^{d}}|f(x-y)|^{p} d_{N} x\right] d_{N} y \\
= & \int_{\mathbb{R}^{d}}|g(y)|^{p}\||f|\|_{p}^{p} d_{N} y \\
= & \||f|\|_{p}^{p} \int_{\mathbb{R}^{d}}|g(y)|^{p} d_{N} y \\
= & \||f|\|_{p}^{p} \cdot\|g \mid\|_{p}^{p} .
\end{aligned}
$$

Raising both sides of this inequality to the power $1 / p$, we get:

$$
\begin{aligned}
& {\left[\int_{\mathbb{R}^{d}}\left|\left[\Gamma\left(\frac{1}{\sqrt{u}}\right) \varphi \diamond \Gamma\left(\frac{1}{\sqrt{v}}\right) \psi\right]\left(\frac{x}{\sqrt{u v}}\right)\right|^{p} e^{-\frac{\langle x, x\rangle}{2 u v}} d_{N} x\right]^{1 / p} } \\
\leq & \||f|\|_{p} \cdot\||g|\|_{p} \\
= & {\left[\int_{\mathbf{R}^{d}}\left|\varphi\left(\frac{x}{\sqrt{v}}\right)\right|^{p} e^{-\frac{\langle x, x\rangle}{2 v}} d_{N} x\right]^{1 / p} \cdot\left[\int_{\mathbf{R}^{d}}\left|\psi\left(\frac{x}{\sqrt{u}}\right)\right|^{p} e^{-\frac{\langle x, x\rangle}{2 u}} d_{N} x\right]^{1 / p} . }
\end{aligned}
$$

Making now the changes of variable: $x_{1}:=(1 / \sqrt{u v}) x$ in the integral

$$
\int_{\mathbb{R}^{d}}\left|\left[\Gamma\left(\frac{1}{\sqrt{u}}\right) \varphi \diamond \Gamma\left(\frac{1}{\sqrt{v}}\right) \psi\right]\left(\frac{x}{\sqrt{u v}}\right)\right|^{p} e^{-\frac{\langle x, x\rangle}{2 u v}} d_{N} x,
$$

$x^{\prime}:=(1 / \sqrt{v}) x$ and $x^{\prime \prime}:=(1 / \sqrt{u}) x$ in the integrals:

$$
\int_{\mathbf{R}^{d}}\left|\varphi\left(\frac{x}{\sqrt{v}}\right)\right|^{p} e^{-\frac{\langle x, x\rangle}{2 v}} d_{N} x
$$

and

$$
\int_{\mathbf{R}^{d}}\left|\psi\left(\frac{x}{\sqrt{u}}\right)\right|^{p} e^{-\frac{\langle x, x\rangle}{2 u}} d_{N} x
$$


respectively, and dividing both sides by $(u v)^{d /(2 p)}$, since $d \mu=\exp (-\langle x, x\rangle / 2) d_{N} x$, we get:

$$
\left\|\Gamma\left(\frac{1}{\sqrt{u}}\right) \varphi \diamond \Gamma\left(\frac{1}{\sqrt{v}}\right) \psi\right\|_{p} \leq\|\varphi\|_{p} \cdot\|\psi\|_{p} .
$$

Theorem 4.2 (General Hölder inequality for Gaussian Wick products.) Let $H$ be a separable Gaussian Hilbert space, $p \in[1, \infty]$, and $u$ and $v$ positive numbers, such that $(1 / u)+(1 / v)=1$. Let $\mathcal{F}(H)$ be the sigma-algebra generated by the random variables $h$ from $H$. Then for any $\varphi$ and $\psi$ in $L^{p}(\Omega, \mathcal{F}(H), P), \Gamma(1 / \sqrt{u}) \varphi \diamond \Gamma(1 / \sqrt{v}) \psi \in L^{p}(\Omega, \mathcal{F}(H), P)$ and the following inequality holds:

$$
\left\|\Gamma\left(\frac{1}{\sqrt{u}}\right) \varphi \diamond \Gamma\left(\frac{1}{\sqrt{v}}\right) \psi\right\|_{p} \leq\|\varphi\|_{p} \cdot\|\psi\|_{p} .
$$

Proof. Let $\left\{e_{n}\right\}_{n \geq 1}$ be an orthonormal basis of $H$. Then $\left\{e_{n}\right\}_{n \geq 1}$ is a set of independent, normally distributed random variables with mean 0 and variance 1 . For every $d \geq 1$, let $\mathcal{F}_{d}$ denote the sigma-algebra generated by the random variables $e_{1}, e_{2}, \ldots, e_{d}$. It is wellknown that every function $f$ from $L^{p}(\Omega, \mathcal{F}(H), P)$ can be approximated in the $p$ norm by a sequence of functions $f_{n}$ from $L^{p}\left(\mathcal{F}_{n}\right):=L^{p}\left(\Omega, \mathcal{F}_{n}, P\right), n \geq 1$. This is due to the fact that the sigma-algebra $\mathcal{F}(H)$ is generated by the cylinder sets, and every cylinder set is in one of the sigma-algebras $\mathcal{F}_{d}$, for some $d \geq 1$. There exist two sequences $\left\{\varphi_{n}\right\}_{n \geq 1}$ and $\left\{\psi_{n}\right\}_{n \geq 1}$ contained in $\cup_{n \geq 1} L^{p}\left(\mathcal{F}_{n}\right)$, such that $\left\|\varphi_{n}-\varphi\right\|_{p} \rightarrow 0$ and $\left\|\psi_{n}-\psi\right\|_{p} \rightarrow 0$, as $n \rightarrow \infty$. These facts imply two things: first $\left\|\varphi_{n}\right\|_{p} \rightarrow\|\varphi\|_{p}$ and $\left\|\psi_{n}\right\|_{p} \rightarrow\|\psi\|_{p}$, as $n \rightarrow \infty$, and second $\varphi_{n} \rightarrow \varphi$ and $\psi_{n} \rightarrow \psi$, in $L^{1}$, as $n \rightarrow \infty$. We know from [9] that $\Gamma(1 / \sqrt{u}) \varphi_{n} \diamond \Gamma(1 / \sqrt{v}) \psi_{n} \rightarrow \Gamma(1 / \sqrt{u}) \varphi \diamond \Gamma(1 / \sqrt{v}) \psi$, in $L^{1}$, as $n \rightarrow \infty$. Since $L^{1}$ convergence implies almost sure convergence for a subsequence, working eventually with a subsequence, we may assume that $\Gamma(1 / \sqrt{u}) \varphi_{n} \diamond \Gamma(1 / \sqrt{v}) \psi_{n} \rightarrow \Gamma(1 / \sqrt{u}) \varphi \diamond \Gamma(1 / \sqrt{v}) \psi$, almost surely, as $n \rightarrow \infty$. Using now Fatou's lemma and the finite dimensional inequality proven in the previous theorem, we have:

$$
\begin{aligned}
\left\|\Gamma\left(\frac{1}{\sqrt{u}}\right) \varphi \diamond \Gamma\left(\frac{1}{\sqrt{v}}\right) \psi\right\|_{p}^{p} & =E\left[\left|\Gamma\left(\frac{1}{\sqrt{u}}\right) \varphi \diamond \Gamma\left(\frac{1}{\sqrt{v}}\right) \psi\right|^{p}\right] \\
& =E\left[\liminf _{n \rightarrow \infty}\left\{\left|\Gamma\left(\frac{1}{\sqrt{u}}\right) \varphi_{n} \diamond \Gamma\left(\frac{1}{\sqrt{v}}\right) \psi_{n}\right|^{p}\right\}\right] \\
& \leq \liminf _{n \rightarrow \infty} E\left[\left|\Gamma\left(\frac{1}{\sqrt{u}}\right) \varphi_{n} \diamond \Gamma\left(\frac{1}{\sqrt{v}}\right) \psi_{n}\right|^{p}\right] \\
& \leq \liminf _{n \rightarrow \infty}\left\{E\left[\left|\varphi_{n}\right|^{p}\right] \cdot E\left[\left|\psi_{n}\right|^{p}\right]\right\} . \\
& =E\left[|\varphi|^{p}\right] \cdot E\left[|\psi|^{p}\right] \\
& =\|\varphi\|_{p}^{p} \cdot\|\psi\|_{p}^{p} .
\end{aligned}
$$

We are now presenting an interesting connection between the Young inequality with the best constant, and Nelson hypercontractivity. The Young inequality with the best constant is the following: 
Theorem 4.3 Let $p, q, r \geq 1$, such that $(1 / p)+(1 / q)=(1 / r)+1$. There exists a constant $C_{p, q, r ; d}>0$, such that, for any $f \in L^{p}\left(\mathbb{R}^{d}, d x\right)$ and $g \in L^{q}\left(\mathbb{R}^{d}, d x\right)$, we have:

$$
\left[\int_{\mathbb{R}^{d}}|(f \star g)(x)|^{r} d x\right]^{1 / r} \leq C_{p, q, r ; d}\left[\int_{\mathbb{R}^{d}}|f(x)|^{p} d x\right]^{1 / p} \cdot\left[\int_{\mathbb{R}^{d}}|g(x)|^{q} d x\right]^{1 / q} .
$$

The sharp constant $C_{p, q, r ; d}$ equals $\left(C_{p} C_{q} / C_{r}\right)^{d}$, where $C_{k}^{2}=k^{1 / k} / k^{\prime 1 / k^{\prime}}$, for any $k \geq 1$, where $k^{\prime}$ is the conjugate of $k$.

See [1], 2], or [11] (Theorem 4.2) for a proof. Let us make the observation that if we replace the Lebesgue measure $d x$, on $\mathbb{R}^{d}$, by $c d x$, where $c$ is any positive constant, then, by convoluting with respect to $c d x$, the best constant $C_{p, q, r: d}$ from inequality (4.3) does not change. This is due to the fact that the left-hand side of (4.3) is multiplied by $c$. $c^{1 / r}=c^{1+(1 / r)}$, while the right-hand side must be multiplied by $c^{1 / p} \cdot c^{1 / q}=c^{(1 / p)+(1 / q)}$, and fortunately $1+(1 / r)=(1 / p)+(1 / q)$. Thus, the inequality (4.3) remains valid, with the same sharp constant $C_{p, q, r ; d}$, even for the normalized Lebesgue measure $d_{N} x$.

Theorem 4.4 Let $d$ be a fixed natural number. Let $p$ and $r$ be positive numbers such that $1<p \leq r$. Then for any $\varphi$ in $L^{p}\left(\mathbb{R}^{d}, \mu\right)$ and any $\psi$ in $L^{\infty}\left(\mathbb{R}^{d}, \mu\right), \Gamma(\sqrt{p-1} / \sqrt{r-1}) \varphi \diamond$ $\Gamma(\sqrt{r-p} / \sqrt{r-1}) \psi$ belongs to $L^{r}\left(\mathbb{R}^{d}, \mu\right)$ and the following inequality holds:

$$
\left\|\left[\Gamma\left(\frac{\sqrt{p-1}}{\sqrt{r-1}}\right) \varphi\right] \diamond\left[\Gamma\left(\frac{\sqrt{r-p}}{\sqrt{r-1}}\right) \psi\right]\right\|_{r} \leq\|\varphi\|_{p} \cdot\|\psi\|_{\infty} .
$$

In particular, if we choose $\psi=1$ (the constant random variable equal to 1 ), then we get the classic Nelson hypercontractivity inequality:

$$
\left\|\Gamma\left(\frac{\sqrt{p-1}}{\sqrt{r-1}}\right) \varphi\right\|_{r} \leq\|\varphi\|_{p}
$$

Proof. Let $u:=(r-1) /(p-1)$ and $v:=(r-1) /(r-p)$. Then we have $(1 / u)+(1 / v)=1$. Let $p^{\prime}$ and $r^{\prime}$ be the conjugates of $p$ and $r$, respectively . Let's go back to the identity (3.1) and multiply both sides of that relation by $\exp \left(-\langle x, x\rangle /\left(2 u v r^{\prime}\right)\right)$. We get:

$$
\begin{aligned}
& {\left[\Gamma\left(\frac{1}{\sqrt{u}}\right) \varphi \diamond \Gamma\left(\frac{1}{\sqrt{v}}\right) \psi\right]\left(\frac{x}{\sqrt{u v}}\right) e^{-\frac{\langle x, x\rangle}{2 u v}\left(1-\frac{1}{r^{\prime}}\right)} } \\
= & e^{\frac{\langle x, x\rangle}{2^{\prime} u v}}\left\{\left[\varphi\left(\frac{x}{\sqrt{v}}\right) e^{-\frac{\langle x, x\rangle}{2 v}}\right] \star\left[\psi\left(\frac{x}{\sqrt{u}}\right) e^{-\frac{\langle x, x\rangle}{2 u}}\right]\right\} \\
= & \int_{\mathbb{R}^{d}}\left[\varphi\left(\frac{x-y}{\sqrt{v}}\right) e^{-\frac{\langle x-y, x-y\rangle}{2 p v}}\right] e^{-\frac{\langle x-y, x-y\rangle}{2 p^{\prime} v}}\left[\psi\left(\frac{y}{\sqrt{u}}\right) e^{-\frac{\langle y, y\rangle}{2 u}}\right] \cdot e^{\frac{\langle x, x\rangle}{2 r^{\prime} u v}} d_{N} y .
\end{aligned}
$$


Let $f(x):=\varphi(x / \sqrt{v}) \exp (-\langle x, x\rangle /(2 p v))$. We have:

$$
\begin{aligned}
& \mid\left[\Gamma\left(\frac{1}{\sqrt{u}}\right) \varphi \diamond \Gamma\left(\frac{1}{\sqrt{v}}\right) \psi\right]\left(\frac{x}{\sqrt{u v}}\right) e^{-\frac{\langle x, x\rangle}{2 u v}\left(1-\frac{1}{r^{\prime}}\right) \mid} \\
\leq & \int_{\mathbb{R}^{d}}|f(x-y)| e^{-\frac{\langle x-y, x-y\rangle}{2 p^{\prime} v}}\left|\psi\left(\frac{y}{\sqrt{u}}\right)\right| e^{-\frac{\langle y, y\rangle}{2 u}} \cdot e^{\frac{\langle x, x\rangle}{2 r^{\prime} u v}} d_{N} y \\
\leq & \int_{\mathbb{R}^{d}}|f(x-y)| e^{-\frac{\langle x-y, x-y\rangle}{2 p^{\prime} v}}\|\psi\|_{\infty} e^{-\frac{\langle y, y\rangle}{2 u}} \cdot e^{\frac{\langle x, x\rangle}{2 r^{\prime} u v}} d_{N} y \\
= & \|\psi\|_{\infty} \int_{\mathbb{R}^{d}}|f(x-y)| \cdot e^{-\frac{\langle x-y, x-y\rangle}{2 p^{\prime} v}} \cdot e^{-\frac{\langle y, y\rangle}{2 u}} \cdot e^{\frac{\langle x, x\rangle}{2 r^{\prime} u v}} d_{N} y \\
= & \|\psi\|_{\infty} \int_{\mathbb{R}^{d}}|f(x-y)| \cdot e^{-\left[\frac{\langle x-y, x-y\rangle}{2 p^{\prime} v}+\frac{\langle y, y\rangle}{2 u}-\frac{\langle x, x\rangle}{2 r^{\prime} u v}\right]} d_{N} y .
\end{aligned}
$$

Let us observe that the expression:

$$
E(x, y)=\frac{\langle x-y, x-y\rangle}{2 p^{\prime} v}+\frac{\langle y, y\rangle}{2 u}-\frac{\langle x, x\rangle}{2 r^{\prime} u v}
$$

is a perfect square. Indeed, the coefficient of $\langle x, x\rangle$ in $E(x, y)$ is:

$$
\begin{aligned}
a & =\frac{1}{2 p^{\prime} v}-\frac{1}{2 r^{\prime} u v} \\
& =\frac{1}{2 v}\left(\frac{1}{p^{\prime}}-\frac{1}{r^{\prime}} \cdot \frac{1}{u}\right) \\
& =\frac{1}{2 v}\left(\frac{p-1}{p}-\frac{r-1}{r} \cdot \frac{p-1}{r-1}\right) \\
& =\frac{p-1}{2 v}\left(\frac{1}{p}-\frac{1}{r}\right) \\
& =\frac{p-1}{2 v} \cdot \frac{r-p}{p r} \\
& =\frac{p-1}{p} \cdot \frac{r-1}{r} \cdot \frac{r-p}{r-1} \cdot \frac{1}{2 v} \\
& =\frac{1}{p^{\prime}} \cdot \frac{1}{r^{\prime}} \cdot \frac{1}{v} \cdot \frac{1}{2 v} \\
& =\frac{1}{2 p^{\prime} r^{\prime} v^{2}} .
\end{aligned}
$$


The coefficient of $\langle y, y\rangle$ in $E(x, y)$ is:

$$
\begin{aligned}
c & =\frac{1}{2 u}+\frac{1}{2 p^{\prime} v} \\
& =\frac{1}{2}\left(\frac{1}{u}+\frac{1}{p^{\prime}} \cdot \frac{1}{v}\right) \\
& =\frac{1}{2}\left(\frac{p-1}{r-1}+\frac{p-1}{p} \cdot \frac{r-p}{r-1}\right) \\
& =\frac{p-1}{2(r-1)}\left(1+\frac{r-p}{p}\right) \\
& =\frac{p-1}{2(r-1)} \cdot \frac{r}{p} \\
& =\frac{1}{2} \cdot \frac{r}{r-1} \cdot \frac{p-1}{p} \\
& =\frac{1}{2} \cdot r^{\prime} \cdot \frac{1}{p^{\prime}} \\
& =\frac{r^{\prime}}{2 p^{\prime}} .
\end{aligned}
$$

The coefficient of $\langle x, y\rangle$ is $E(x, y)$ is:

$$
b=-\frac{1}{p^{\prime} v} .
$$

Thus we have:

$$
\begin{aligned}
E(x, y) & =a\langle x, x\rangle+b\langle x, y\rangle+c\langle y, y\rangle \\
& =\frac{1}{2 p^{\prime} r^{\prime} v^{2}}\langle x, x\rangle-\frac{1}{p^{\prime} v}\langle x, y\rangle+\frac{r^{\prime}}{2 p^{\prime}}\langle y, y\rangle \\
& =\frac{1}{2 p^{\prime} r^{\prime} v^{2}}\left\langle x-r^{\prime} v y, x-r^{\prime} v y\right\rangle .
\end{aligned}
$$

It follows now from (4.6) that:

$$
\begin{aligned}
& \left|\left[\Gamma\left(\frac{1}{\sqrt{u}}\right) \varphi \diamond \Gamma\left(\frac{1}{\sqrt{v}}\right) \psi\right]\left(\frac{x}{\sqrt{u v}}\right) e^{-\frac{\langle x, x\rangle}{2 u v} \cdot \frac{1}{r}}\right| \\
\leq & \|\psi\|_{\infty} \int_{\mathbb{R}^{d}}|f(x-y)| \cdot e^{-\frac{1}{2 p^{\prime} r^{\prime} v^{2}}\left\langle x-r^{\prime} v y, x-r^{\prime} v y\right\rangle} d_{N} y .
\end{aligned}
$$

Let us make the change of variable $t:=x-y$ in the last integral. We obtain:

$$
\begin{aligned}
& \mid\left[\Gamma\left(\frac{1}{\sqrt{u}}\right) \varphi \diamond \Gamma\left(\frac{1}{\sqrt{v}}\right) \psi\right]\left(\frac{x}{\sqrt{u v}}\right) e^{-\frac{\langle x, x\rangle}{2 u v r} \mid} \\
\leq & \|\psi\|_{\infty} \int_{\mathbb{R}^{d}}|f(t)| \cdot e^{-\frac{1}{2 p^{\prime} r^{\prime} v^{2}}\left\langle x-r^{\prime} v(x-t), x-r^{\prime} v(x-t)\right\rangle} d_{N} t \\
= & \|\psi\|_{\infty} \int_{\mathbb{R}^{d}}|f(t)| \cdot e^{-\frac{1}{2 p^{\prime} r^{\prime} v^{2}}\left\langle\left(1-r^{\prime} v\right) x+r^{\prime} v t,\left(1-r^{\prime} v\right) x+r^{\prime} v t\right\rangle} d_{N} t \\
= & \|\psi\|_{\infty} \int_{\mathbb{R}^{d}}|f(t)| \cdot e^{-\frac{1}{2 p^{\prime} r^{\prime} v^{2}} \cdot\left(-r^{\prime} v\right)^{2}\left\langle\frac{r^{\prime} v-1}{r^{\prime} v} x-t, \frac{r^{\prime} v-1}{r^{\prime} v} x-t\right\rangle} d_{N} t \\
= & \|\psi\|_{\infty} \int_{\mathbb{R}^{d}}|f(t)| \cdot e^{-\frac{r^{\prime}}{2 p^{\prime}} \cdot\left\langle\frac{1}{s^{\prime}} x-t, \frac{1}{s^{\prime}} x-t\right\rangle} d_{N} t,
\end{aligned}
$$


where $s:=r^{\prime} v$ and $s^{\prime}$ is the conjugate of $s$. Let us observe that the last integral is a convolution product. Indeed, if we define $g(x):=\exp \left(-\left[r^{\prime} /\left(2 p^{\prime}\right)\right] \cdot\langle x, x\rangle\right)$, then:

$$
\begin{aligned}
& \mid\left[\Gamma\left(\frac{1}{\sqrt{u}}\right) \varphi \diamond \Gamma\left(\frac{1}{\sqrt{v}}\right) \psi\right]\left(\frac{x}{\sqrt{u v}}\right) e^{-\frac{\langle x, x\rangle}{2 u v r} \mid} \\
\leq & \|\psi\|_{\infty} \int_{\mathbb{R}^{d}}|f(t)| \cdot e^{-\frac{r^{\prime}}{2 p^{\prime}} \cdot\left\langle\frac{1}{s^{\prime}} x-t, \frac{1}{s^{\prime}} x-t\right\rangle} d_{N} t \\
= & \|\psi\|_{\infty} \cdot[f \star g]\left(\frac{1}{s^{\prime}} x\right),
\end{aligned}
$$

for all $x \in \mathbb{R}^{d}$. Replacing $x$ by $s^{\prime} x$, in the last inequality, we obtain:

$$
\left|\left[\Gamma\left(\frac{1}{\sqrt{u}}\right) \varphi \diamond \Gamma\left(\frac{1}{\sqrt{v}}\right) \psi\right]\left(\frac{s^{\prime} x}{\sqrt{u v}}\right) e^{-\frac{s^{\prime 2}\langle x, x\rangle}{2 u v r}}\right| \leq\|\psi\|_{\infty} \cdot[f \star g](x),
$$

for all $x \in \mathbb{R}^{d}$. Raising this inequality to the power $r$ and integrating with respect to the normalized Lebesgue measure $d_{N} x$, we get:

$$
\begin{aligned}
& \left\{\int_{\mathbb{R}^{d}}\left|\left[\Gamma\left(\frac{1}{\sqrt{u}}\right) \varphi \diamond \Gamma\left(\frac{1}{\sqrt{v}}\right) \psi\right]\left(\frac{s^{\prime} x}{\sqrt{u v}}\right) e^{-\frac{s^{\prime 2}\langle x, x\rangle}{2 u v r}}\right|^{r} d_{N} x\right\}^{1 / r} \\
\leq & \|\psi\|_{\infty} \cdot\left\{\int_{\mathbb{R}^{d}}|(f \star g)(x)|^{r} d_{N} x\right\}^{1 / r} .
\end{aligned}
$$

Making, the change of variable $x^{\prime}:=\left(s^{\prime} / \sqrt{u v}\right) x$ in the integral from the left, we obtain:

$$
\left(\frac{\sqrt{u v}}{s^{\prime}}\right)^{d / r}\left\|\left|\left[\Gamma\left(\frac{1}{\sqrt{u}}\right) \varphi \diamond \Gamma\left(\frac{1}{\sqrt{v}}\right) \psi\right] \cdot e^{-\frac{1}{2 r}\langle\cdot, \cdot\rangle}\right|\right\|_{r} \leq\|\psi\|_{\infty} \cdot\||f \star g|\|_{r} .
$$

The left hand--side is a Gaussian $L^{r}$-norm, and so, we get:

$$
\left(\frac{\sqrt{u v}}{s^{\prime}}\right)^{d / r}\left\|\Gamma\left(\frac{1}{\sqrt{u}}\right) \varphi \diamond \Gamma\left(\frac{1}{\sqrt{v}}\right) \psi\right\|_{r} \leq\|\psi\|_{\infty} \cdot\||f \star g|\|_{r} .
$$

Since $r \geq p,(1 / r)+1-(1 / p) \leq 1$. Thus there exists $q \geq 1$, such that $(1 / r)+1=(1 / p)+(1 / q)$. We apply now the Young inequality with the sharp constant, in the right side of (4.9), and obtain:

$$
\begin{aligned}
\left\|\Gamma\left(\frac{1}{\sqrt{u}}\right) \varphi \diamond \Gamma\left(\frac{1}{\sqrt{v}}\right) \psi\right\|_{r} & \leq\left(\frac{s^{\prime}}{\sqrt{u v}}\right)^{d / r} \cdot\|\psi\|_{\infty} \cdot\||f \star g|\|_{r} \\
& \leq\left(\frac{s^{\prime}}{\sqrt{u v}}\right)^{d / r} \cdot\|\psi\|_{\infty} \cdot\left(C_{p} C_{q} / C_{r}\right)^{d}\||f|\|_{p} \cdot\|g \mid\|_{q}
\end{aligned}
$$

where $C_{p}^{2}=p^{1 / p} / p^{\prime 1 / p^{\prime}}$. Since $f(x)=\varphi(x / \sqrt{v}) \exp (-\langle x, x\rangle /(2 p v))$, it is easy to see that:

$$
\||f|\|_{p}=(\sqrt{v})^{d / p}\|\varphi\|_{p}
$$

Because $g(x):=\exp \left(-\left[r^{\prime} /\left(2 p^{\prime}\right)\right] \cdot\langle x, x\rangle\right)$, it is not hard to see that:

$$
\||g|\|_{q}=\left(\sqrt{\frac{p^{\prime}}{q r^{\prime}}}\right)^{d / q}
$$


Thus, inequality (4.10) becomes:

$$
\left\|\Gamma\left(\frac{1}{\sqrt{u}}\right) \varphi \diamond \Gamma\left(\frac{1}{\sqrt{v}}\right) \psi\right\|_{r} \leq\left(\frac{s^{\prime}}{\sqrt{u v}}\right)^{d / r}\left(C_{p} C_{q} / C_{r}\right)^{d}(\sqrt{v})^{d / p}\left(\sqrt{\frac{p^{\prime}}{q r^{\prime}}}\right)^{d / q}\|\varphi\|_{p}\|\psi\|_{\infty} .
$$

Therefore, to prove (4.4), we just need to show that:

$$
\left(\frac{s^{\prime}}{\sqrt{u v}}\right)^{d / r} \cdot\left(C_{p} C_{q} / C_{r}\right)^{d} \cdot(\sqrt{v})^{d / p} \cdot\left(\sqrt{\frac{p^{\prime}}{q r^{\prime}}}\right)^{d / q}=1
$$

which (by raising both sides to the power $2 / d$ ) is equivalent to:

$$
\frac{C_{p}^{2} C_{q}^{2} s^{\prime 2 / r} p^{1 / q} v^{1 / p-1 / r}}{C_{r}^{2} u^{1 / r} q^{1 / q} r^{1 / q}}=1
$$

Since $1 / p-1 / r=1-1 / q$ and $1-1 / q=1 / q^{\prime}$, we have to prove that:

$$
\frac{C_{p}^{2} C_{q}^{2} s^{2 / r} p^{1 / q} v^{1 / q^{\prime}}}{C_{r}^{2} u^{1 / r} q^{1 / q} r^{1 / q}}=1
$$

Let:

$$
C:=\frac{C_{p}^{2} C_{q}^{2} s^{2 / r} p^{1 / q} v^{1 / q^{\prime}}}{C_{r}^{2} u^{1 / r} q^{1 / q} r^{\prime 1 / q}}
$$

To prove (4.13) we will write $u, v$, and $s^{\prime}$ in terms of $p, q, r$ and their conjugates. We have:

$$
\begin{aligned}
u & =\frac{r-1}{p-1} \\
& =\frac{r}{p} \cdot \frac{1-\frac{1}{r}}{1-\frac{1}{p}} \\
& =\frac{r}{p} \cdot \frac{\frac{1}{r^{\prime}}}{\frac{1}{p^{\prime}}} \\
& =\frac{r p^{\prime}}{p r^{\prime}} .
\end{aligned}
$$

We also have:

$$
\begin{aligned}
v & =\frac{r-1}{r-p} \\
& =\frac{r\left(1-\frac{1}{r}\right)}{p r\left(\frac{1}{p}-\frac{1}{r}\right)} \\
& =\frac{1}{p} \cdot \frac{1-\frac{1}{r}}{\frac{1}{p}-\frac{1}{r}} .
\end{aligned}
$$


Let's remember that $(1 / p)-(1 / r)=1 / q^{\prime}$. Thus, we obtain:

$$
\begin{aligned}
v & =\frac{1}{p} \cdot \frac{1-\frac{1}{r}}{\frac{1}{p}-\frac{1}{r}} \\
& =\frac{1}{p} \cdot \frac{\frac{1}{r^{\prime}}}{\frac{1}{q^{\prime}}} \\
& =\frac{q^{\prime}}{p r^{\prime}} .
\end{aligned}
$$

Finally, we have:

$$
\begin{aligned}
s^{\prime} & =\frac{s}{s-1} \\
& =\frac{r^{\prime} v}{r^{\prime} v-1} .
\end{aligned}
$$

Replacing $v$ by $q^{\prime} /\left(p r^{\prime}\right)$ we get:

$$
\begin{aligned}
s^{\prime} & =\frac{r^{\prime} v}{r^{\prime} v-1} \\
& =\frac{r^{\prime} \frac{q^{\prime}}{p r^{\prime}}}{r^{\prime} \frac{q^{\prime}}{p r^{\prime}}-1} \\
& =\frac{\frac{q^{\prime}}{p}}{\frac{q^{\prime}}{p}-1} .
\end{aligned}
$$

Dividing both the numerator and denominator of the last fraction by $q^{\prime}$ we get:

$$
\begin{aligned}
s^{\prime} & =\frac{\frac{1}{p}}{\frac{1}{p}-\frac{1}{q^{\prime}}} \\
& =\frac{\frac{1}{p}}{\frac{1}{p}-\left(1-\frac{1}{q}\right)} \\
& =\frac{\frac{1}{p}}{\frac{1}{p}+\frac{1}{q}-1} \\
& =\frac{\frac{1}{p}}{\frac{1}{r}} \\
& =\frac{r}{p} .
\end{aligned}
$$

Let us substitute $C_{p}^{2}, C_{q}^{2}, C_{r}^{2}, u, v$, and $s^{\prime}$, in the formula (4.14). We have:

$$
\begin{aligned}
C & =\frac{C_{p}^{2} C_{q}^{2} s^{\prime 2 / r} p^{1 / q} v^{1 / q^{\prime}}}{C_{r}^{2} u^{1 / r} q^{1 / q} r^{\prime 1 / q}} \\
& =\frac{\frac{p^{1 / p}}{p^{\prime 1 / p^{\prime}}} \cdot \frac{q^{1 / q}}{q^{\prime 1 / q^{\prime}}} \cdot\left(\frac{r}{p}\right)^{2 / r} \cdot p^{1 / q} \cdot\left(\frac{q^{\prime}}{p r^{\prime}}\right)^{1 / q^{\prime}}}{\frac{r^{1 / r}}{r^{\prime 1 / r^{\prime}}} \cdot\left(\frac{r p^{\prime}}{p r^{\prime}}\right)^{1 / r} \cdot q^{1 / q} \cdot r^{\prime 1 / q}} .
\end{aligned}
$$


Observe that the factors $q^{1 / q}, q^{1 / q^{\prime}}$, and $r^{2 / r}$ cancel. Collecting the powers of $p, p^{\prime}$, and $r^{\prime}$, we obtain:

$$
\begin{aligned}
& C=p^{\frac{1}{p}-\frac{1}{r}-\frac{1}{q^{\prime}}} \cdot p^{\prime \frac{1}{q}-\frac{1}{p^{\prime}}-\frac{1}{r}} \cdot r^{\prime \frac{1}{r^{\prime}}+\frac{1}{r}-\frac{1}{q^{\prime}}-\frac{1}{q}} \\
& =p^{\frac{1}{p}-\frac{1}{r}-\left(1-\frac{1}{q}\right)} \cdot p^{\prime \frac{1}{q}-\left(1-\frac{1}{p}\right)-\frac{1}{r}} \cdot r^{\prime\left(\frac{1}{r^{\prime}}+\frac{1}{r}\right)-\left(\frac{1}{q^{\prime}}+\frac{1}{q}\right)} \\
& =p^{\left(\frac{1}{p}+\frac{1}{q}\right)-\left(\frac{1}{r}+1\right)} \cdot p^{\left(\frac{1}{p}+\frac{1}{q}\right)-\left(\frac{1}{r}+1\right)} \cdot r^{1-1} \\
& =p^{0} \cdot p^{\prime 0} \cdot r^{\prime 0} \\
& =1 \text {. }
\end{aligned}
$$

Passing from the finite dimensional case to the infinite dimensional case, can be done in the same way as before, using Fatou's lemma.

We now present a more general Hölder inequality. The proof of this inequality uses the following theorem of Lieb (see [10] or [11] (page 100)).

Theorem 4.5 Fix $k>1$, integers $n_{1}, \ldots, n_{k}$ and numbers $p_{1}, \ldots, p_{k} \geq 1$. Let $M \geq 1$ and let $B_{i}$ (for $i=1, \ldots, k$ ) be a linear mapping from $\mathbb{R}^{M}$ to $\mathbb{R}^{n_{i}}$. Let $Z: \mathbb{R}^{M} \rightarrow \mathbb{R}^{+}$be some fixed Gaussian function,

$$
Z(x)=\exp [-\langle x, J x\rangle]
$$

with $J$ a real, positive-semidefinite $M \times M$ matrix (possible zero).

For functions $f_{i}$ in $L^{p_{i}}\left(\mathbb{R}^{n_{i}}\right)$ consider the integral $I_{Z}$ and the number $C_{Z}$ :

$$
\begin{gathered}
I_{Z}\left(f_{1}, \ldots, f_{k}\right)=\int_{\mathbb{R}^{M}} Z(x) \prod_{i=1}^{k} f_{i}\left(B_{i} x\right) d x \\
C_{Z}:=\sup \left\{I_{Z}\left(f_{1}, \ldots, f_{k}\right)\left|\left\|\left|f_{i}\right|\right\|_{p_{i}}=1 \text { for } i=1, \ldots, k\right\} .\right.
\end{gathered}
$$

Then $C_{Z}$ is determined by restricting the $f$ 's to be Gaussian functions, i.e.,

$$
\begin{aligned}
C_{Z}= & \sup \left\{I _ { Z } ( f _ { 1 } , \ldots , f _ { k } ) \left|\left\|\left|f_{i}\right|\right\|_{p_{i}}=1 \text { and } f_{i}(x)=c_{i} \exp \left[-\left\langle x, J_{i} x\right\rangle\right]\right.\right. \\
& \text { with } \left.c_{i}>0, \text { and } J_{i} \text { a real, symmetric, positive -definite } n_{i} \times n_{i} \text { matrix }\right\} .
\end{aligned}
$$

Corollary 4.6 Let $p, q, r \geq 1$. Let $B_{1}$ and $B_{2}$ be linear maps from $\mathbb{R}^{2}$ to $\mathbb{R}^{2}$, and $J$ a real, positive-semidefinite $2 \times 2$ matrix (possible zero). For $f$ in $L^{p}\left(\mathbb{R}^{2}\right)$ and $g$ in $L^{q}\left(\mathbb{R}^{2}\right)$, we consider the product:

$$
\left(f \star_{B_{1}, B_{2}, J} g\right)(x)=\int_{\mathbb{R}} f\left(B_{1}(x, y)\right) g\left(B_{2}(x, y)\right) e^{-\langle(x, y), J(x, y)\rangle} d_{N} y .
$$

We define:

$$
C:=\sup \left\{\left\|\left|f \star_{B_{1}, B_{2}, J} g\right|\right\|_{r}\left|\||f|\|_{p}=\||g|\|_{q}=1\right\} .\right.
$$

Then $C$ is determined by restricting $f$ and $g$ to be Gaussian functions. 
Proof. Let $r^{\prime}$ be the conjugate of $r$. For any $k \geq 1$, we denote by $\mathcal{G}_{k}$ the set of Gaussian functions of $L^{k}$-norm equal to 1 . Using the duality between $L^{r}$ and $L^{r^{\prime}}$, Lieb's theorem, and Hölder inequality, we have:

$$
\begin{aligned}
C & =\sup \left\{\left\|\left|f \star_{B_{1}, B_{2}, J} g\right|\right\|_{r}\left|\||f|\|_{p}=\||g|\|_{q}=1\right\}\right. \\
= & \sup \left\{\left|\int_{\mathbb{R}}\left(f \star_{B_{1}, B_{2}, J} g\right)(x) h(x) d_{N} x\right|\left|\||f|\|_{p}=\||g|\|_{q}=\||h|\|_{r^{\prime}}=1\right\}\right. \\
= & \sup \left\{\left|\int_{\mathbb{R}} \int_{\mathbb{R}} f\left(B_{1}(x, y)\right) g\left(B_{2}(x, y)\right) h(x) e^{-\langle(x, y), J(x, y)\rangle} d_{N} x d_{N} y\right| \mid\right. \\
& \left.\left\||| f\left|\left\|_{p}=\right\|\right| g\left|\left\|_{q}=\right\|\right| h \mid\right\|_{r^{\prime}}=1\right\} \\
\leq & \sup \left\{\left|\int_{\mathbb{R}} \int_{\mathbb{R}} f\left(B_{1}(x, y)\right) g\left(B_{2}(x, y)\right) h(x) e^{-\langle(x, y), J(x, y)\rangle} d_{N} x d_{N} y\right| \mid f \in \mathcal{G}_{p}, g \in \mathcal{G}_{q}, h \in \mathcal{G}_{r^{\prime}}\right\} \\
\leq & \sup \left\{\left\|\left|f \star_{B_{1}, B_{2}, J} g\right|\right\|_{r} \cdot\||h|\|_{r^{\prime}} \mid f \in \mathcal{G}_{p}, g \in \mathcal{G}_{q}, h \in \mathcal{G}_{r^{\prime}}\right\} \\
& =\sup \left\{\left\|\left|f \star_{B_{1}, B_{2}, J} g\right|\right\|_{r} \cdot 1 \mid f \in \mathcal{G}_{p}, g \in \mathcal{G}_{q}\right\} .
\end{aligned}
$$

Theorem 4.7 (Full Hölder inequality for Gaussian Hilbert spaces.) Let $H$ be $a$ separable Gaussian Hilbert space. Let $u, v, p, q$, and $r$ be numbers greater than 1, such that:

$$
\frac{1}{u}+\frac{1}{v}=1
$$

and

$$
\frac{1}{u(p-1)}+\frac{1}{v(q-1)}=\frac{1}{r-1}
$$

Then for all $\varphi$ in $L^{p}(\Omega, \mathcal{F}(H), P)$ and $\psi$ in $L^{q}(\Omega, \mathcal{F}(H), P), \Gamma(1 / \sqrt{u}) \varphi \diamond \Gamma(1 / \sqrt{v}) \psi$ belongs to $L^{r}(\Omega, \mathcal{F}(H), P)$ and the following inequality holds:

$$
\|\Gamma(1 / \sqrt{u}) \varphi \diamond \Gamma(1 / \sqrt{v}) \psi\|_{r} \leq\|\varphi\|_{p} \cdot\|\psi\|_{q} .
$$

Proof. Let $p^{\prime}, q^{\prime}$, and $r^{\prime}$ be the conjugates of $p, q$, and $r$, respectively. Since:

$$
\begin{aligned}
\frac{1}{p-1} & =\frac{p}{p-1}-1 \\
& =p^{\prime}-1
\end{aligned}
$$

and similarly $1 /(q-1)=q^{\prime}-1$, and $1 /(r-1)=r^{\prime}-1$, condition (4.22) is equivalent to:

$$
\begin{aligned}
r^{\prime}-1 & =\frac{1}{r-1} \\
& =\frac{1}{u(p-1)}+\frac{1}{v(q-1)} \\
& =\frac{1}{u}\left(p^{\prime}-1\right)+\frac{1}{v}\left(q^{\prime}-1\right) \\
& =\frac{1}{u} \cdot p^{\prime}+\frac{1}{v} \cdot q^{\prime}-\left(\frac{1}{u}+\frac{1}{v}\right) \\
& =\frac{1}{u} \cdot p^{\prime}+\frac{1}{v} \cdot q^{\prime}-1 .
\end{aligned}
$$


That means, we have:

$$
\frac{p^{\prime}}{u}+\frac{q^{\prime}}{v}=r^{\prime}
$$

Following the same steps as before, it is enough to check the inequality in the finite dimensional case. Multiplying both sides of the convolution identity (3.1) by $\exp \left(1 /\left(2 u v r^{\prime}\right)\langle x, x\rangle\right)$, we obtain:

$$
\begin{aligned}
& {\left[\Gamma\left(\frac{1}{\sqrt{u}}\right) \varphi \diamond \Gamma\left(\frac{1}{\sqrt{v}}\right) \psi\right]\left(\frac{x}{\sqrt{u v}}\right) e^{-\frac{\langle x, x\rangle}{2 u v}\left(1-\frac{1}{r^{\prime}}\right)} } \\
= & e^{\frac{\langle x, x\rangle}{2 r^{\prime} u v}}\left\{\left[\varphi\left(\frac{x}{\sqrt{v}}\right) e^{\left.\left.-\frac{\langle x, x\rangle}{2 v}\right] \star\left[\psi\left(\frac{x}{\sqrt{u}}\right) e^{-\frac{\langle x, x\rangle}{2 u}}\right]\right\}}\right.\right. \\
= & \int_{\mathbb{R}^{d}}\left[\varphi\left(\frac{x-y}{\sqrt{v}}\right) e^{-\frac{\langle x-y, x-y\rangle}{2 p v}}\right] e^{-\frac{\langle x-y, x-y\rangle}{2 p^{\prime} v}}\left[\psi\left(\frac{y}{\sqrt{u}}\right) e^{-\frac{\langle y, y\rangle}{2 q u}}\right] e^{-\frac{\langle y, y\rangle}{2 q^{\prime} u}} \cdot e^{\frac{\langle x, x\rangle}{2 r^{\prime} u v}} d_{N} y .
\end{aligned}
$$

Let $f(x):=\varphi(x / \sqrt{v}) \exp (-\langle x, x\rangle /(2 p v))$ and $g(x):=\psi(x / \sqrt{u}) \exp (-\langle x, x\rangle /(2 q u))$. We have:

$$
\begin{aligned}
& \mid\left[\Gamma\left(\frac{1}{\sqrt{u}}\right) \varphi \diamond \Gamma\left(\frac{1}{\sqrt{v}}\right) \psi\right]\left(\frac{x}{\sqrt{u v}}\right) e^{-\frac{\langle x, x\rangle}{2 u v} \cdot \frac{1}{r} \mid} \\
\leq & \int_{\mathbb{R}^{d}}|f(x-y)| e^{-\frac{\langle x-y, x-y\rangle}{2 p^{\prime} v}}|g(y)| e^{-\frac{\langle y, y\rangle}{2 q^{\prime} u}} \cdot e^{\frac{\langle x, x\rangle}{2 r^{\prime} u v}} d_{N} y \\
= & \int_{\mathbb{R}^{d}}|f(x-y)| \cdot|g(y)| \cdot e^{-\frac{\langle x-y, x-y\rangle}{2 p^{\prime} v}-\frac{\langle y, y\rangle}{2 q^{\prime} u}+\frac{\langle x, x\rangle}{2 r^{\prime} u v}} d_{N} y .
\end{aligned}
$$

As before, we are now showing that the expression:

$$
E(x, y)=\frac{\langle x-y, x-y\rangle}{2 p^{\prime} v}+\frac{\langle y, y\rangle}{2 q^{\prime} u}-\frac{\langle x, x\rangle}{2 r^{\prime} u v}
$$

is a perfect square. Indeed, the coefficient of $\langle x, x\rangle$ in $E(x, y)$ is:

$$
\begin{aligned}
a & =\frac{1}{2 p^{\prime} v}-\frac{1}{2 r^{\prime} u v} \\
& =\frac{1}{2 p^{\prime} r^{\prime} v}\left(r^{\prime}-\frac{p^{\prime}}{u}\right) \\
\text { from (4.24) } & =\frac{1}{2 p^{\prime} r^{\prime} v} \cdot \frac{q^{\prime}}{v} \\
& =\frac{1}{2 p^{\prime} q^{\prime} r^{\prime}} \cdot\left(\frac{q^{\prime}}{v}\right)^{2} .
\end{aligned}
$$

The coefficient of $\langle y, y\rangle$ in $E(x, y)$ is:

$$
\begin{aligned}
c & =\frac{1}{2 q^{\prime} u}+\frac{1}{2 p^{\prime} v} \\
& =\frac{1}{2 p^{\prime} q^{\prime}}\left(\frac{p^{\prime}}{u}+\frac{q^{\prime}}{v}\right) \\
\text { from (4.24) } & =\frac{1}{2 p^{\prime} q^{\prime}} \cdot r^{\prime} \\
& =\frac{1}{2 p^{\prime} q^{\prime} r^{\prime}} \cdot r^{\prime 2} .
\end{aligned}
$$


The coefficient of $\langle x, y\rangle$ is $E(x, y)$ is:

$$
\begin{aligned}
b & =-\frac{1}{p^{\prime} v} \\
& =-\frac{1}{p^{\prime} q^{\prime} r^{\prime}} \cdot\left(\frac{q^{\prime}}{v} \cdot r^{\prime}\right) .
\end{aligned}
$$

Thus we have:

$$
\begin{aligned}
E(x, y) & =a\langle x, x\rangle+b\langle x, y\rangle+c\langle y, y\rangle \\
& =\frac{1}{2 p^{\prime} q^{\prime} r^{\prime}}\left[\left(\frac{q^{\prime}}{v}\right)^{2}\langle x, x\rangle-2\left(\frac{q^{\prime}}{v} \cdot r^{\prime}\right)\langle x, y\rangle+r^{\prime 2}\langle y, y\rangle\right] \\
& =\frac{1}{2 p^{\prime} q^{\prime} r^{\prime}}\left\langle\frac{q^{\prime}}{v} x-r^{\prime} y, \frac{q^{\prime}}{v} x-r^{\prime} y\right\rangle .
\end{aligned}
$$

It follows now from (4.25) that:

$$
\begin{aligned}
& \mid\left[\Gamma\left(\frac{1}{\sqrt{u}}\right) \varphi \diamond \Gamma\left(\frac{1}{\sqrt{v}}\right) \psi\right]\left(\frac{x}{\sqrt{u v}}\right) e^{-\frac{\langle x, x\rangle}{2 u v r} \mid} \\
\leq & \int_{\mathbb{R}^{d}}|f(x-y)| \cdot|g(y)| \cdot e^{-\frac{1}{2 p^{\prime} q^{\prime} r^{\prime}}\left\langle\frac{q^{\prime}}{v} x-r^{\prime} y, \frac{q^{\prime}}{v} x-r^{\prime} y\right\rangle} d_{N} y .
\end{aligned}
$$

Claim 1: For all $d \geq 1, f \in L^{p}\left(\mathbb{R}^{d}, d_{N} x\right)$, and $g \in L^{q}\left(\mathbb{R}^{d}, d_{N} y\right)$, we have:

$$
\left\{\int_{\mathbb{R}^{d}}\left[\int_{\mathbb{R}^{d}}|f(x-y)| \cdot|g(y)| \cdot J_{d}(x, y) d_{N} y\right]^{r} d_{N} x\right\}^{1 / r} \leq C^{d}\||f|\|_{p} \cdot\||g|\|_{q},
$$

where:

$$
J_{d}(x, y)=e^{-\frac{1}{2 p^{\prime} q^{\prime} r^{\prime}}\left\langle\frac{q^{\prime}}{v} x-r^{\prime} y, \frac{q^{\prime}}{v} x-r^{\prime} y\right\rangle}
$$

and

$$
C^{2}=v^{\frac{1}{r}-\frac{1}{p}} u^{\frac{1}{r}-\frac{1}{q}}
$$

To prove this claim, we reduce the problem to the one-dimensional case, via Minkowski's inequality, copying the argument from [11] (page 201). Namely, let us assume that (4.27) holds for two dimensions $d_{1}=m$ and $d_{2}=n$. We can prove that (4.27) holds for $d=m+n$, using Minkowski's inequality in the form in which the discrete summation is replaced by the continuous integration, in the following way. Let $x=\left(x_{m}, x_{n}\right)$ be a generic vector in $\mathbb{R}^{m+n}$, where $x_{m}$ and $x_{n}$ are generic vectors in $\mathbb{R}^{m}$ and $\mathbb{R}^{n}$, respectively. Let us observe that, for all $x=\left(x_{m}, x_{n}\right)$ and $y=\left(y_{m}, y_{n}\right)$ in $\mathbb{R}^{m+n}$, we have:

$$
J_{m+n}(x, y)=J_{m}\left(x_{m}, y_{m}\right) \cdot J_{n}\left(x_{n}, y_{n}\right) .
$$


We have:

$$
\begin{aligned}
& \left\{\int_{\mathbb{R}^{m+n}}\left[\int_{\mathbb{R}^{m+n}}|f(x-y)| \cdot|g(y)| \cdot J_{m+n}(x, y) d_{N} y\right]^{r} d_{N} x\right\}^{1 / r} \\
= & \left\{\int _ { \mathbb { R } ^ { m } } \int _ { \mathbb { R } ^ { n } } \left[\int_{\mathbb{R}^{m}} \int_{\mathbb{R}^{n}}\left|f\left(x_{m}-y_{m}, x_{n}-y_{n}\right)\right| \cdot\left|g\left(y_{m}, y_{n}\right)\right|\right.\right. \\
\leq & \left\{\int _ { \mathbb { R } ^ { m } } \left\{\int _ { \mathbb { R } ^ { m } } J _ { m } ( x _ { m } , y _ { m } ) \left[\int _ { \mathbb { R } ^ { n } } \left(\int_{\mathbb{R}^{n}}\left|f\left(x_{m}-y_{m}, x_{n}-y_{n}\right)\right| \cdot\left|g\left(y_{m}, y_{n}\right)\right|\right.\right.\right.\right. \\
\leq & \left.\left.\left.\left\{\int_{\mathbb{R}^{m}}\left[\int_{\mathbb{R}^{m}} J_{m}\left(x_{m}, y_{m}\right) C^{n} \| \mid f\left(x_{n}, y_{n}\right) d_{N} y_{n}\right)^{r} d_{N} x_{n}\right]^{1 / r} d_{N} y_{m}\right\}^{r} d_{N} x_{m}\right\}^{1 / r}\left(\left\|_{p} \cdot\right\|\left|g\left(y_{m}, \cdot\right)\right| \|_{q} d_{N} y_{m}\right]^{r} d_{N} x_{m}\right\}^{1 / r} \\
= & C^{n}\left\{\int_{\mathbb{R}^{m}}\left[\int_{\mathbb{R}^{m}} J_{m}\left(x_{m}, y_{m}\right)\left\|\left|f\left(x_{m}-y_{m}, \cdot\right)\right|\right\|_{p} \cdot\left\|\left|g\left(y_{m}, \cdot\right)\right|\right\|_{q} d_{N} y_{m}\right]^{r} d_{N} x_{m}\right\}^{1 / r} \\
\leq & C^{n} \cdot C^{m}\||f|\|_{p} \cdot\||g|\|_{q} \\
= & C^{m+n}\||f|\|_{p} \cdot\||g|\|_{q} .
\end{aligned}
$$

This shows that in order to prove (4.27), it is enough to prove it for the dimension $d=1$ only. To achieve this, since the function $(x, y) \mapsto\left[\left(q^{\prime} / v\right) x-r^{\prime} y\right]^{2}$ is non-negative, according to Lieb theorem, it is enough to check it for exponential functions of the form $f(x)=c_{1} \exp \left[-(s / 2) x^{2}\right]$ and $g(x)=c_{2} \exp \left[-(t / 2) x^{2}\right]$, where $s>0, t>0$, and $c_{1}$ and $c_{2}$ are positive constants chosen such that $\||f|\|_{p}=\||g|\|_{q}=1$. Let us first compute the values of $c_{1}$ and $c_{2}$. We have:

$$
\begin{aligned}
\||f|\|_{p} & =c_{1}\left[\int_{\mathbb{R}} e^{-\frac{p s}{2} x^{2}} d_{N} x\right]^{1 / p} \\
\left(\text { let } x^{\prime}:=\sqrt{p s} x\right) & =c_{1}\left[\frac{1}{\sqrt{p s}} \int_{\mathbb{R}} e^{-\frac{x^{\prime 2}}{2}} d_{N} x^{\prime}\right]^{1 / p} \\
& =c_{1} \frac{1}{(\sqrt{p s})^{1 / p}} .
\end{aligned}
$$

Thus, in order to have $\||f|\|_{p}=1$, we must have:

$$
c_{1}=(\sqrt{p s})^{\frac{1}{p}} .
$$

Similarly, in order to have $\|\mid g\|_{q}=1$, we must have:

$$
c_{2}=(\sqrt{q t})^{\frac{1}{q}} .
$$

Hence, we have:

$$
\begin{aligned}
& \left\|\int_{\mathbb{R}} f(\cdot-y) g(y) e^{-\left(\frac{q^{\prime}}{v} \cdot-r^{\prime} y\right)^{2}} d_{N} y \mid\right\|_{r} \\
= & (\sqrt{p s})^{1 / p}(\sqrt{q t})^{1 / q}\left\{\int_{\mathbb{R}}\left[\int_{\mathbb{R}} e^{-\frac{s}{2}(x-y)^{2}} e^{-\frac{t}{2} y^{2}} e^{-\frac{1}{2 p^{\prime} q^{\prime} r^{\prime}}\left(\frac{q^{\prime}}{v} x-r^{\prime} y\right)^{2}} d_{N} y\right]^{r} d_{N} x\right\}^{1 / r} .
\end{aligned}
$$


Let $\alpha:=q^{\prime} /\left(v \sqrt{p^{\prime} q^{\prime} r^{\prime}}\right), \beta:=r^{\prime} / \sqrt{p^{\prime} q^{\prime} r^{\prime}}$, and $\gamma:=p^{\prime} /\left(u \sqrt{p^{\prime} q^{\prime} r^{\prime}}\right)$. Let us observe first that $\alpha+\gamma=\beta$, since $\left(p^{\prime} / u\right)+\left(q^{\prime} / v\right)=r^{\prime}$. We have:

$$
\begin{aligned}
& \left\|\int_{\mathbb{R}} f(\cdot-y) g(y) e^{-\left(\frac{q^{\prime}}{v} \cdot-r^{\prime} y\right)^{2}} d_{N} y \mid\right\|_{r} \\
= & (\sqrt{p s})^{1 / p}(\sqrt{q t})^{1 / q}\left\{\int_{\mathbb{R}}\left[\int_{\mathbb{R}} e^{-\frac{s}{2}(x-y)^{2}} e^{-\frac{t}{2} y^{2}} e^{-\frac{1}{2}(\alpha x-\beta y)^{2}} d_{N} y\right]^{r} d_{N} x\right\}^{1 / r} \\
= & (\sqrt{p s})^{1 / p}(\sqrt{q t})^{1 / q}\left\{\int_{\mathbb{R}}\left[e^{-\frac{\left(s+\alpha^{2}\right)}{2} x^{2}} \int_{\mathbb{R}} e^{-\frac{\left(s+t+\beta^{2}\right)}{2} y^{2}+(s+\alpha \beta) x y} d_{N} y\right]^{r} d_{N} x\right\}^{1 / r} .
\end{aligned}
$$

In the last integral we make the change of variable $y^{\prime}=\sqrt{s+t+\beta^{2}} \cdot y$. Completing the square, we obtain:

$$
\begin{aligned}
& \left\|\left|\int_{\mathbb{R}} f(\cdot-y) g(y) e^{-\left(\frac{q^{\prime}}{v} \cdot-r^{\prime} y\right)^{2}} d_{N} y\right|\right\|_{r} \\
= & (\sqrt{p s})^{1 / p}(\sqrt{q t})^{1 / q}\left\{\int_{\mathbb{R}}\left[e^{-\frac{\left(s+\alpha^{2}\right)}{2} x^{2}} \frac{1}{\sqrt{s+t+\beta^{2}}} \int_{\mathbb{R}} e^{-\frac{1}{2} y^{2}+\frac{s+\alpha \beta}{\sqrt{s+t+\beta^{2}}} x y} d_{N} y\right]^{r} d_{N} x\right\}^{1 / r} \\
= & \sqrt{\frac{(p s)^{1 / p}(q t)^{1 / q}}{s+t+\beta^{2}}}\left\{\int_{\mathbb{R}}\left[e^{-\frac{\left(s+\alpha^{2}\right)}{2} x^{2}} \cdot e^{\frac{(s+\alpha \beta)^{2}}{2\left(s+t+\beta^{2}\right)} x^{2}} \int_{\mathbb{R}} e^{-\frac{1}{2}\left(y-\frac{s+\alpha \beta}{\sqrt{s+t+\beta^{2}}} x\right)^{2}} d_{N} y\right]^{r} d_{N} x\right\}^{1 / r} .
\end{aligned}
$$

Therefore,

$$
\begin{aligned}
& \left\|\left|\int_{\mathbb{R}} f(\cdot-y) g(y) e^{-\left(\frac{q^{\prime}}{v} \cdot-r^{\prime} y\right)^{2}} d_{N} y\right|\right\|_{r} \\
& =\sqrt{\frac{(p s)^{1 / p}(q t)^{1 / q}}{s+t+\beta^{2}}}\left\{\int_{\mathbb{R}}\left[e^{-\frac{\left(s+\alpha^{2}\right)}{2} x^{2}+\frac{(s+\alpha \beta)^{2}}{2\left(s+t+\beta^{2}\right)} x^{2}}\right]^{r} d_{N} x\right\}^{1 / r} \\
& =\sqrt{\frac{(p s)^{1 / p}(q t)^{1 / q}}{s+t+\beta^{2}}}\left\{\int_{\mathbb{R}}\left[e^{-\frac{\left(s+\alpha^{2}\right)\left(s+t+\beta^{2}\right)+(s+\alpha \beta)^{2}}{2\left(s+t+\beta^{2}\right)} x^{2}}\right]^{r} d_{N} x\right\}^{1 / r} \\
& =\sqrt{\frac{(p s)^{1 / p}(q t)^{1 / q}}{s+t+\beta^{2}}}\left\{\int_{\mathbb{R}}\left[e^{-\frac{\left[s(\beta-\alpha)^{2}+t \alpha^{2}+s t\right]}{2\left(s+t+\beta^{2}\right)} x^{2}}\right]^{r} d_{N} x\right\}^{1 / r} \\
& =\sqrt{\frac{(p s)^{1 / p}(q t)^{1 / q}}{s+t+\beta^{2}}}\left\{\int_{\mathbb{R}} e^{-\frac{r\left(s \gamma^{2}+t \alpha^{2}+s t\right)}{2\left(s+t+\beta^{2}\right)} x^{2}} d_{N} x\right\}^{1 / r} \\
& =\sqrt{\frac{(p s)^{1 / p}(q t)^{1 / q}}{s+t+\beta^{2}}} \cdot \sqrt{\frac{\left(s+t+\beta^{2}\right)^{1 / r}}{r^{1 / r}\left(s \gamma^{2}+t \alpha^{2}+s t\right)^{1 / r}}} \\
& =\sqrt{\frac{p^{1 / p} q^{1 / q}}{r^{1 / r}}} \cdot \sqrt{\frac{s^{1 / p} t^{1 / q}}{\left(s+t+\beta^{2}\right)^{1 / r^{\prime}}\left(\gamma^{2} s+\alpha^{2} t+s t\right)^{1 / r}}} .
\end{aligned}
$$

To finish our proof we need to show that:

$$
\sup _{s>0, t>0}\left\{\frac{p^{1 / p} q^{1 / q}}{r^{1 / r}} \cdot \frac{s^{1 / p} t^{1 / q}}{\left(s+t+\beta^{2}\right)^{1 / r^{\prime}}\left(\gamma^{2} s+\alpha^{2} t+s t\right)^{1 / r}}\right\}=v^{\frac{1}{r}-\frac{1}{p}} u^{\frac{1}{r}-\frac{1}{q}} .
$$


Before we compute this supremum, we would like to outline the intuition behind what we are going to do next. Let us observe that the numerator $s^{1 / p} t^{1 / q}$, being a product, is somehow like a geometric mean, while the factors from the denominator $\left(s+t+\beta^{2}\right)$ and $\left(\gamma^{2} s+\alpha^{2} t+s t\right)$, being sums, are like arithmetic means. We know from the inequality between the geometric and arithmetic means of positive numbers, that the geometric mean is always dominated by the arithmetic mean, and this classic inequality is based on the concavity of the logarithmic function.

Let $S:=(p v) s$ and $T:=(q u) t$. We have:

$$
\begin{aligned}
& \frac{p^{1 / p} q^{1 / q}}{r^{1 / r}} \cdot \frac{s^{1 / p} t^{1 / q}}{\left(s+t+\beta^{2}\right)^{1 / r^{\prime}}\left(\gamma^{2} s+\alpha^{2} t+s t\right)^{1 / r}} \\
= & \frac{p^{1 / p} q^{1 / q}}{r^{1 / r}} \cdot \frac{\frac{1}{(p v)^{1 / p}} S^{1 / p} \frac{1}{(q u)^{1 / q}} T^{1 / q}}{\left(\frac{1}{p v} \cdot S+\frac{1}{q u} \cdot T+\beta^{2} \cdot 1\right)^{1 / r^{\prime}}\left(\frac{\gamma^{2}}{p v} \cdot S+\frac{\alpha^{2}}{q u} \cdot T+\frac{1}{p q u v} \cdot S T\right)^{1 / r}} \\
= & \frac{1}{v^{1 / p} u^{1 / q} r^{1 / r}} \cdot \frac{S^{1 / p} T^{1 / q}}{\left(\frac{1}{p v} \cdot S+\frac{1}{q u} \cdot T+\beta^{2} \cdot 1\right)^{1 / r^{\prime}}\left(\frac{\gamma^{2}}{p v} \cdot S+\frac{\alpha^{2}}{q u} \cdot T+\frac{1}{p q u v} \cdot S T\right)^{1 / r}}
\end{aligned}
$$

Let us observe that:

$$
\frac{1}{p v}+\frac{1}{q u}+\beta^{2}=1
$$

Indeed, we have:

$$
\begin{aligned}
\frac{1}{p v}+\frac{1}{q u}+\beta^{2} & =\frac{1}{p v}+\frac{1}{q u}+\frac{r^{\prime}}{p^{\prime} q^{\prime}} \\
& =\frac{1}{p v}+\frac{1}{q u}+\frac{\frac{p^{\prime}}{u}+\frac{q^{\prime}}{v}}{p^{\prime} q^{\prime}} \\
& =\frac{1}{p v}+\frac{1}{q u}+\frac{1}{q^{\prime} u}+\frac{1}{p^{\prime} v} \\
& =\frac{1}{v}\left(\frac{1}{p}+\frac{1}{p^{\prime}}\right)+\frac{1}{u}\left(\frac{1}{q}+\frac{1}{q^{\prime}}\right) \\
& =\frac{1}{v} \cdot 1+\frac{1}{u} \cdot 1 \\
& =1 .
\end{aligned}
$$

Let us also observe that:

$$
\frac{\gamma^{2}}{p v}+\frac{\alpha^{2}}{q u}+\frac{1}{p q u v}=\frac{1}{u v r}
$$


Indeed, we have:

$$
\begin{aligned}
& \frac{\gamma^{2}}{p v}+\frac{\alpha^{2}}{q u}+\frac{1}{p q u v} \\
= & \frac{p^{\prime}}{u^{2} q^{\prime} r^{\prime}} \cdot \frac{1}{p v}+\frac{q^{\prime}}{v^{2} p^{\prime} r^{\prime}} \cdot \frac{1}{q u}+\frac{1}{p q u v} \\
= & \frac{1}{u v r^{\prime}}\left(\frac{p^{\prime}}{p u q^{\prime}}+\frac{q^{\prime}}{q v p^{\prime}}+\frac{1}{p q} \cdot r^{\prime}\right) \\
= & \frac{1}{u v r^{\prime}}\left[\frac{p^{\prime}}{p u q^{\prime}}+\frac{q^{\prime}}{q v p^{\prime}}+\frac{1}{p q} \cdot\left(\frac{p^{\prime}}{u}+\frac{q^{\prime}}{v}\right)\right] \\
= & \frac{1}{u v r^{\prime}}\left[\frac{p^{\prime}}{p u q^{\prime}}+\frac{q^{\prime}}{q v p^{\prime}}+\frac{p^{\prime}}{p q u}+\frac{q^{\prime}}{p q u}\right] \\
= & \frac{1}{u v r^{\prime}}\left[\frac{p^{\prime}}{p u}\left(\frac{1}{q^{\prime}}+\frac{1}{q}\right)+\frac{q^{\prime}}{q v}\left(\frac{1}{p^{\prime}}+\frac{1}{p}\right)\right] \\
= & \frac{1}{u v r^{\prime}}\left[\frac{p^{\prime}}{p u} \cdot 1+\frac{q^{\prime}}{q v} \cdot 1\right] \\
= & \frac{1}{u v r^{\prime}}\left[\frac{p /(p-1)}{p u}+\frac{q /(q-1)}{q v}\right] \\
= & \frac{1}{u v r^{\prime}}\left[\frac{1}{u(p-1)}+\frac{1}{v(q-1)}\right] \\
= & \frac{1}{u v r /(r-1)} \cdot \frac{1}{r-1} \\
= & \frac{1}{u v r} .
\end{aligned}
$$

We go back to the denominator of formula (4.34) and apply the Jensen inequality for the strictly concave downward function $L(x)=\ln (x)$. From (4.35) we conclude that:

$$
\begin{aligned}
\ln \left(\frac{1}{p v} \cdot S+\frac{1}{q u} \cdot T+\beta^{2} \cdot 1\right) & \geq \frac{1}{p v} \ln (S)+\frac{1}{q u} \ln (T)+\beta^{2} \ln (1) \\
& =\ln \left(S^{1 /(p v)} T^{1 /(q u)}\right) .
\end{aligned}
$$

Exponentiating both sides of this inequality and then rasing them to the power $1 / r^{\prime}$, we obtain:

$$
\left(\frac{1}{p v} \cdot S+\frac{1}{q v} \cdot T+\beta^{2} \cdot 1\right)^{1 / r^{\prime}} \geq S^{1 /\left(p v r^{\prime}\right)} T^{1 /\left(q u r^{\prime}\right)} .
$$

Formula (4.36) shows that in order to obtain a convex combination in the sum:

$$
\frac{\gamma^{2}}{p v} \cdot S+\frac{\alpha^{2}}{q u} \cdot T+\frac{1}{p q u v} \cdot S T
$$

we need first to multiply it by $K:=u v r$. After doing this, applying again the strict concavity of the function ln, we obtain:

$$
\begin{aligned}
\ln \left(K \frac{\gamma^{2}}{p v} \cdot S+K \frac{\alpha^{2}}{q u} \cdot T+K \frac{1}{p q u v} \cdot S T\right) & \geq K \frac{\gamma^{2}}{p v} \ln (S)+K \frac{\alpha^{2}}{q u} \ln (T)+K \frac{1}{p q u v} \ln (S T) \\
& =\ln \left(S^{\left.\frac{K \gamma^{2}}{p v}+\frac{K}{p q u v} T^{\frac{K \alpha^{2}}{q u}+\frac{K}{p q u v}}\right) .}\right.
\end{aligned}
$$


This inequality is equivalent to:

$$
\begin{aligned}
\left(\frac{\gamma^{2}}{p v} \cdot S+\frac{\alpha^{2}}{q u} \cdot T+\frac{1}{p q u v} \cdot S T\right)^{1 / r} & \geq \frac{1}{K^{1 / r}} S^{\frac{K \gamma^{2}}{p v r}+\frac{K}{p q u v r}} T^{\frac{K \alpha^{2}}{q u r}+\frac{K}{p q u v r}} \\
& =\frac{1}{(u v r)^{1 / r}} S^{\frac{u \gamma^{2}}{p}+\frac{1}{p q}} T^{\frac{v \alpha^{2}}{q}+\frac{1}{p q}} .
\end{aligned}
$$

Going now back to the formula (4.34) and using the inequalities (4.37) and (4.38), we obtain:

$$
\begin{aligned}
& \frac{p^{1 / p} q^{1 / q}}{r^{1 / r}} \cdot \frac{s^{1 / p} t^{1 / q}}{\left(s+t+\beta^{2}\right)^{1 / r^{\prime}}\left(\gamma^{2} s+\alpha^{2} t+s t\right)^{1 / r}} \\
= & \frac{1}{v^{1 / p} u^{1 / q} r^{1 / r}} \cdot \frac{S^{1 / p} T^{1 / q}}{\left(\frac{1}{p v} \cdot S+\frac{1}{q v} \cdot T+\beta^{2} \cdot 1\right)^{1 / r^{\prime}}\left(\frac{\gamma^{2}}{p v} \cdot S+\frac{\alpha^{2}}{q u} \cdot T+\frac{1}{p q u v} \cdot S T\right)^{1 / r}} \\
\leq & \frac{1}{v^{1 / p} u^{1 / q} r^{1 / r}} \cdot \frac{S^{1 / p} T^{1 / q}}{S^{1 /\left(p v r^{\prime}\right)} T^{1 /\left(q u r^{\prime}\right)} \frac{1}{(u v r)^{1 / r}} S^{\left(u \gamma^{2}\right) / p+1 /(p q)} T^{\left(v \alpha^{2}\right) / q+1 /(p q)}} .
\end{aligned}
$$

The exponent of $S$ in the denominator of the last fraction is:

$$
\begin{aligned}
\frac{1}{p v r^{\prime}}+\frac{p^{\prime}}{u p q^{\prime} r^{\prime}}+\frac{1}{p q} & =\frac{1}{p r^{\prime}}\left[\frac{1}{v}+\frac{p^{\prime}}{u q^{\prime}}+\frac{1}{q} \cdot r^{\prime}\right] \\
& =\frac{1}{p r^{\prime}}\left[\frac{1}{v}+\frac{p^{\prime}}{u q^{\prime}}+\frac{1}{q}\left(\frac{p^{\prime}}{u}+\frac{q^{\prime}}{v}\right)\right] \\
& =\frac{1}{p r^{\prime}}\left[\frac{1}{v}+\frac{p^{\prime}}{u}\left(\frac{1}{q^{\prime}}+\frac{1}{q}\right)+\frac{q^{\prime}}{v} \cdot \frac{1}{q}\right] \\
& =\frac{1}{p r^{\prime}}\left[\frac{1}{v}+\frac{p^{\prime}}{u} \cdot 1+\frac{q^{\prime}}{v}\left(1-\frac{1}{q^{\prime}}\right)\right] \\
& =\frac{1}{p r^{\prime}}\left[\frac{1}{v}+\frac{p^{\prime}}{u}+\frac{q^{\prime}}{v}-\frac{1}{v}\right] \\
& =\frac{1}{p r^{\prime}} \cdot r^{\prime} \\
& =\frac{1}{p} .
\end{aligned}
$$

Similarly, the exponent of $T$ in the denominator of the same fraction is $1 / q$. Hence, for all $s, t>0$, we have:

$$
\begin{aligned}
\frac{p^{1 / p} q^{1 / q}}{r^{1 / r}} \cdot \frac{s^{1 / p} t^{1 / q}}{\left(s+t+\beta^{2}\right)^{1 / r^{\prime}}\left(\gamma^{2} s+\alpha^{2} t+s t\right)^{1 / r}} & \leq v^{(1 / r)-(1 / p)} u^{(1 / r)-(1 / q)} \cdot \frac{S^{1 / p} T^{1 / q}}{S^{1 / p} T^{1 / q}} \\
& =v^{(1 / r)-(1 / p)} u^{(1 / r)-(1 / q)} .
\end{aligned}
$$

The equality in (4.39) holds if and only if $S=T=1$, due to the strict concavity of the function $y=\ln (x)$. This is equivalent to $s=1 /(p v)$ and $t=1 /(q u)$, since $S=(p v) s$ and $T=(q u) t$. Thus, we have:

$$
\sup _{s>0, t>0}\left\{\frac{p^{1 / p} q^{1 / q}}{r^{1 / r}} \cdot \frac{s^{1 / p} t^{1 / q}}{\left(s+t+\beta^{2}\right)^{1 / r^{\prime}}\left(\gamma^{2} s+\alpha^{2} t+s t\right)^{1 / r}}\right\}=v^{(1 / r)-(1 / p)} u^{(1 / r)-(1 / q)} .
$$


Going back to the inequality (4.26), we conclude that:

$$
\begin{aligned}
& \left\{\int_{\mathbb{R}^{d}}\left|\left[\Gamma\left(\frac{1}{\sqrt{u}}\right) \varphi \diamond \Gamma\left(\frac{1}{\sqrt{v}}\right) \psi\right]\left(\frac{x}{\sqrt{u v}}\right) e^{-\frac{\langle x, x\rangle}{2 u v} \cdot \frac{1}{r}}\right|^{r} d_{N} x\right\}^{1 / r} \\
& \leq\left\{\int_{\mathbb{R}^{d}}\left[\int_{\mathbb{R}^{d}}|f(x-y)| \cdot|g(y)| \cdot e^{-\frac{1}{2 p^{\prime} q^{\prime} r^{\prime}}\left\langle\frac{q^{\prime}}{v} x-r^{\prime} y, \frac{q^{\prime}}{v} x-r^{\prime} y\right\rangle} d_{N} y\right]^{r} d_{N} x\right\}^{1 / r} \\
& \leq \sqrt{v^{\frac{d}{r}-\frac{d}{p}} u^{\frac{d}{r}-\frac{d}{q}}}\||f|\|_{p}\||g|\|_{q} \\
& =v^{\frac{d}{2 r}-\frac{d}{2 p}} u^{\frac{d}{2 r}-\frac{d}{2 q}}\left[\int_{\mathbb{R}^{d}}\left|\varphi\left(\frac{x}{\sqrt{v}}\right)\right|^{p} e^{-\frac{\langle x, x\rangle}{2 v}} d_{N} x\right]^{1 / p}\left[\int_{\mathbb{R}^{d}}\left|\psi\left(\frac{x}{\sqrt{u}}\right)\right|^{q} e^{-\frac{\langle x, x\rangle}{2 u}} d_{N} x\right]^{1 / q} .
\end{aligned}
$$

This inequality is equivalent to:

$$
\begin{aligned}
& (u v)^{-\frac{d}{2 r}}\left\{\int_{\mathbb{R}^{d}}\left|\left[\Gamma\left(\frac{1}{\sqrt{u}}\right) \varphi \diamond \Gamma\left(\frac{1}{\sqrt{v}}\right) \psi\right]\left(\frac{x}{\sqrt{u v}}\right)\right|^{r} e^{-\frac{\langle x, x\rangle}{2 u v}} d_{N} x\right\}^{1 / r} \\
\leq & v^{-\frac{d}{2 p}}\left[\int_{\mathbb{R}^{d}}\left|\varphi\left(\frac{x}{\sqrt{v}}\right)\right|^{p} e^{-\frac{\langle x, x\rangle}{2 v}} d_{N} x\right]^{1 / p} u^{-\frac{d}{2 q}}\left[\int_{\mathbb{R}^{d}}\left|\psi\left(\frac{x}{\sqrt{u}}\right)\right|^{q} e^{-\frac{\langle x, x\rangle}{2 u}} d_{N} x\right]^{1 / q} .
\end{aligned}
$$

Making now the changes of variable $x^{\prime}:=x / \sqrt{u v}$ in the left, and $x_{1}:=x / \sqrt{v}$ and $x_{2}:=x / \sqrt{u}$ in the right, and moving back to the Gaussian norms, we obtain:

$$
\left\|\Gamma\left(\frac{1}{\sqrt{u}}\right) \varphi \diamond \Gamma\left(\frac{1}{\sqrt{v}}\right) \psi\right\|_{r} \leq\|\varphi\|_{p} \cdot\|\psi\|_{q} .
$$

To prove the inequality in the infinite dimensional case we proceed in the following way. Let $H$ be a separable Gaussian Hilbert space. Let $\left\{e_{n}\right\}_{n \geq 1}$ be an orthonormal basis of centered Gaussian random variables from $H$. For all $d \geq 1$, let

$$
H_{d}:=\mathbb{C} e_{1} \oplus \mathbb{C} e_{2} \oplus \cdots \oplus \mathbb{C} e_{d}
$$

Let $\mathcal{F}_{d}:=\mathcal{F}\left(H_{d}\right)$, i.e., the smallest sigma-algebra with respect to which $e_{1}, e_{2}, \ldots, e_{d}$ are measurable. If $\varphi \in L^{p}(\Omega, \mathcal{F}(H), P)$ and $\psi \in L^{q}(\Omega, \mathcal{F}(H), P)$, and if we denote the conditional expectations of $\varphi$ and $\psi$, with respect to $\mathcal{F}_{d}$, by $\varphi_{d}$ and $\psi_{d}$, respectively, i.e., $\varphi_{d}:=E\left[\varphi \mid \mathcal{F}_{d}\right]$ and $\psi_{d}:=E\left[\psi \mid \mathcal{F}_{d}\right]$, then it is not hard to see that:

$$
\begin{aligned}
& E\left[\Gamma(1 / \sqrt{u}) \varphi \mid \mathcal{F}_{d}\right]=\Gamma(1 / \sqrt{u}) \varphi_{d}, \\
& E\left[\Gamma(1 / \sqrt{v}) \psi \mid \mathcal{F}_{d}\right]=\Gamma(1 / \sqrt{v}) \psi_{d},
\end{aligned}
$$

and

$$
E\left[\Gamma(1 / \sqrt{u}) \varphi \diamond \Gamma(1 / \sqrt{v}) \psi \mid \mathcal{F}_{d}\right]=\Gamma(1 / \sqrt{u}) \varphi_{d} \diamond \Gamma(1 / \sqrt{v}) \psi_{d}
$$

Since $\left\{\mathcal{F}_{d}\right\}_{d \geq 1}$ is an increasing family of sigma-algebras and the sigma-algebra generated by them is $\mathcal{F}(H)$, using the Martingale Convergence Theorem we conclude that:

$$
E\left[\Gamma(1 / \sqrt{u}) \varphi \diamond \Gamma(1 / \sqrt{v}) \psi \mid \mathcal{F}_{d}\right] \rightarrow \Gamma(1 / \sqrt{u}) \varphi \diamond \Gamma(1 / \sqrt{v}) \psi
$$




$$
E\left[\varphi \mid \mathcal{F}_{d}\right] \rightarrow \varphi
$$

and

$$
E\left[\psi \mid \mathcal{F}_{d}\right] \rightarrow \psi
$$

as $d \rightarrow \infty$, both almost surely and in $L^{1}(\Omega, \mathcal{F}(H), P)$. Using now the fact that the result is true in the finite dimensional case and Fatou's Lemma as before, we conclude that:

$$
\|\Gamma(1 / \sqrt{u}) \varphi \diamond \Gamma(1 / \sqrt{v}) \psi\|_{r} \leq\|\varphi\|_{p} \cdot\|\psi\|_{q}
$$

\section{References}

[1] Beckner, W.: Inequalities in Fourier Analysis, Ann. of Math. 102, 1975, 159-182.

[2] Brascamp, H.J. and Lieb, E.H.: Best constants in Young's inequality, its converse, and its generalization to more than three functions, Adv. in Math. 20, 1976, 151-173.

[3] Carlen, E. and Cordero-Erausquin, D.: Subadditivity of the entropy and its relation to Brascamp-Lieb type inequalities, Geom. Funct. Analysis 19 (2009), 373-405.

[4] Carlen, E., Lieb, E., and Loss, M.: A sharp form of Young's inequality on $S^{N}$ and related entropy inequalities, Jour. Geom. Analysis 14 (2004), 487-520.

[5] Janson, S: Gaussian Hilbert Spaces, Cambridge Tracts in Math. 129: Cambridge University Press, 1997.

[6] Kuo, H.-H.: White Noise Distribution Theory, Probability and Stochastic Series, CRC Press, Inc. 1996.

[7] Kuo, H.-H., Saitô, K., and Stan, A.I.: A Hausdorff-Young inequality for white noise analysis, in Quantum Information IV, T. Hida and K. Saitô, Eds., pp. 115-126, World Scientific, River Edge, NJ, USA, 2002.

[8] Lanconelli, A. and Stan, A.I.: Hölder Type Inequalities for Norms of Wick Products, J. of Appl. Math. Stoch. An., vol. 2008, Article ID 254897, 22 pages, 2008. doi: $10.1155 / 2008 / 254897$.

[9] Lanconelli, A. and Stan, A.I.: Some inequalities for norms of Gaussian Wick products, Stoch. An. Appl., Vol. 28, Issue 3, 2010, 523-539.

[10] Lieb, E.H.: Gaussian kernels have only Gaussian maximizers, Invent. Math. 102, 1990, 179-208.

[11] Lieb, E.H. and Loss, M.: Analysis Second Edition, Graduate Studies in Mathematics, 14, AMS, Providence, Rhode Island, 2001.

[12] Obata, N.: White Noise Calculus on Fock Space, Lecture Notes in Math. 1577, Springer-Verlag, 1994. 